\title{
Moduli and Canonical Forms for Linear Dynamical Systems II: The Topological Case
}

\author{
by \\ Michiel HaZewinkel \\ Econometric Institute \\ Erasmus University, Rotterdam
}

ABSTRACT

In this paper we study real linear dynamical systems $\dot{x}=F x+G u, y=H x, x \in \mathbf{R}^{n}=$ state space, $u \in \mathbf{R}^{m}=$ input space, $y \in \mathbf{R}^{p}=$ output space, under the equivalence relation induced by base change in state space; or in other words we study triples of matrices with real coefficients $(F, G, H)$ of sizes $n \times n, n \times m, p \times n$ respectively, under the action $(F, G, H)$ $\rightarrow\left(T F T^{-1}, T G, H T^{-1}\right)$ of $G L_{n}(\mathbf{R})$, the group of invertible real $n \times n$ matrices. One of the central questions studied is: "do there exist continuous canonical forms for this equivalence relation?". After various trivial obstructions to the existence of such forms have been removed the answer is very roughly: no if $m \geq 2, p \geq 2$, yes if $m=1$, or $p=1$. For a precise statement cf. theorem 1.7.

Existence or nonexistence of continuous canonical forms is related to the existence of a universal family of real linear dynamical systems. More precisely continuous canonical forms exist if such a universal family exists and if the underlying vector bundle of this family is the trivial vector bundle. In the case studied we show that a universal family in the appropriate sense does exist.

The methods used are purely (differential) topological and in particular do not involve any algebraic geometry. There is a corresponding algebraic theory over any field $k$ instead of $\mathbf{R}$ which is the subject of part III of this series of papers.

\section{Introduction}

We consider linear dynamical systems with constant coefficients

$$
\begin{aligned}
& x=F x+G u, x \in \mathbf{R}^{n}, u \in \mathbf{R}^{m} \\
& y=H x, \quad y \in \mathbf{R}^{p},
\end{aligned}
$$

(continuous time), and systems

$$
\begin{array}{lc}
x_{t+1}=F x_{t}+u_{t}, & x_{t} \in \mathbf{R}^{n}, u_{t} \in \mathbf{R}^{m}, t \in \mathbf{Z} \\
y_{t}=H x_{t} & y_{t} \in \mathbf{R}^{p} \\
\text { (discrete time) } &
\end{array}
$$

I.e. there are $m$ inputs, $p$ outputs and the state space dimension is $n$. A change of basis in state space changes the triple of matrices $(F, G, H)$ as follows 
(1.3) $\quad(F, G, H) \mapsto(F, G, H)^{T}=\left(T F T^{-1}, T G, H T^{-1}\right), T \in G L_{n}(\mathbf{R})$

where $G L_{n}(\mathbf{R})$ is the group of invertible $n \times n$ matrices. Motivated by e.g. identification of systems theory, the question now arises whether there exist continuous canonical forms for this action of $G L_{n}(\mathbf{R})$ on the space of all triples ( $F$, $G, H)$. A precise definition of this notion follows. Let $L_{m, n, p}(\mathbf{R})$ denote the topological space of all triples of matrices $(F, G, H)$ of sizes $n \times n, n \times m, p \times n$ respectively; $L_{m, n, p}(\mathbf{R})$ is naturally identifiable with $\mathbf{R}^{(n+m+p) n}$ and is given the corresponding topology.

1.4 Definition. Two elements $(F, G, H),(\bar{F}, \bar{G}, \bar{H}) \in L_{m, n, p}(\mathbf{R})$ are called $G L_{n}(\mathbf{R})$ equivalent if there is a $T \in G L_{n}(\mathbf{R})$ such that $(F, G, H)^{T}=(\bar{F}, \bar{G}, \bar{H})$.

We can now define a continuous canonical form as follows:

1.5 Definition. A continuous canonical form on a subspace $L^{\prime} \subset L_{m, n, p}(\mathbf{R})$ is a continuous map $c: L^{\prime} \rightarrow L^{\prime}$ such that

(1.5.1) $c(F, G, H)$ is $G L_{n}(\mathbf{R})$ equivalent to $(F, G, H)$ for all $(F, G, H) \in L^{\prime}$,

(1.5.2) $c(F, G, H)=c(\bar{F}, \bar{G}, \bar{H})$ if and only if $(F, G, H),(\bar{F}, \bar{G}, \bar{H}) \in L^{\prime}$ are $G L_{n}(\mathbf{R})$ equivalent.

Of course one usually lets $L^{\prime}$ be a $G L_{n}(\mathbf{R})$ invariant subspace of $L_{m, n, p}$, i.e. a subspace such that $(F, G, H) \in L^{\prime} \Rightarrow(F, G, H)^{T} \in L^{\prime}$ for all $T \in G L_{n}(\mathbf{R})$.

One now remarks immediately that, for trivial reasons, there is no continuous canonical form on all of $L_{m, n, p}(\mathbf{R})$; more precisely because there are socalled jump phenomena; that is there are families $\left(F_{t}, G_{t}, H_{t}\right), t \in \mathbf{R}$, of elements in $L_{m, n, p}(\mathbf{R})$, depending continuously on the parameter $t$ such that $\left(F_{t}, G_{t}, H_{t}\right)$ and $\left(F_{s}, G_{s}, H_{s}\right)$ are $G L_{n}(\mathbf{R})$-equivalent for all $t \neq 0, s \neq 0$ but such that $\left(F_{t}, G_{t}, H_{t}\right)$ is not $G L_{n}(\mathbf{R})$-equivalent to $\left(F_{o}, G_{o}, H_{o}\right)$ for $\mathrm{t} \neq 0$. One now easily checks that if $L^{\prime}$ contains such a family then no continuous canonical form on $L^{\prime}$ can exist. One example of such a family is obtained as follows. Let $G_{1}$ be any nonzero matrix, $F_{1}$ any matrix, $H_{1}=0$. Now define $G_{t}=t G_{1}, H_{t}=0, F_{t}=F_{1}$ for all $t \in \mathbf{R}$.

So, for continuous canonical forms to exist we must first of all see to it that no jump phenomena can occur. One subspace of $L_{m, n, p}(\mathbf{R})$ for which this condition is satisfied is the subspace $L_{m, n, p}^{c r}(\mathbf{R})$ of all completely reachable triples $(F, G, H)$. For a definition cf. 2.2 below.

This fits in rather well with the "identification of systems" point of view where one is mainly interested in systems which are completely reachable and completely observable because as far as input/output behaviour is concerned every linear system is equivalent to such a one. We denote with $L_{m, n, p}^{c r, c o}(\mathbf{R})$ the subspace of $L_{m, n, p}(\mathbf{R})$ of completely reachable and completely observable triples $(F, G, H)$; cf. 2.2 below for a definition of completely observable.

In addition to $L_{m, n, p}^{c r, c o}(\mathbf{R})$ we consider some more $\left(G L_{n}(\mathbf{R})\right.$-invariant subspaces of $L_{m, n, p}(\mathbf{R})$. A short list of interesting subspaces might be the following:

1.6 List of Subspaces.

$L_{m, n, p}^{c r}(\mathbf{R})$ : all triples $(F, G H)$ such that $(F, G)$ is completely reachable.

$L_{m, n, p}^{c o}(\mathbf{R})$ : all triples $(F, G, H)$ such that $(F, H)$ is completely observable. 
$L_{m . n . p}^{c r . c o}(\mathbf{R})=L_{m, n, p}^{c r}(\mathbf{R}) \cap L_{m, n, p}^{c o}(\mathbf{R})$.

$L_{m . n . p}^{\lambda}(\mathbf{R})$ : all triples $(F, G, H)$ such that the eigenvalues of $F$ are all real, distinct and different from zero.

$L_{m, n, p}^{c r, c o, \lambda}(\mathbf{R})=L_{m, n, p}^{c r . c o}(\mathbf{R}) \cap L_{m, n, p}^{i}(\mathbf{R})$

$L_{m, n, p}^{\rho}(\mathbf{R})$ : all triples $(F, G, H)$ such that $(F, G)$ is completely reachable, $(F, H)$ is completely observable, $\operatorname{rank}(G)=\min (n, m)$, rank $H=\min (n, p)$.

$L_{m . n, p}^{\rho . \lambda}(\mathbf{R})=L_{m . n . p}^{\rho}(\mathbf{R}) \cap L_{m . n . p}^{\lambda}(\mathbf{R})$

The following theorem then describes the main results of section 3 below.

1.7 THEOREM. The following table lists necessary and sufficient conditions for the existence of a continuous canonical form on various $G L_{n}(\mathbf{R})$-invariant subspaces $L^{\prime}$ of $L_{m, n, p}(\mathbf{R})$

\begin{tabular}{l|c|c} 
& space $L^{\prime}$ & $\begin{array}{c}\text { necessary and sufficient conditions for existence } \\
\text { of a continuous canonical form }\end{array}$ \\
\hline (i) & $L_{m, n, p}^{\text {cr }}(\mathbf{R})$ & $m=1$ \\
\hline (ii) & $L_{m, n, p}^{\text {co }}(\mathbf{R})$ & $p=1$ \\
\hline (iii) & $L_{m, n, p}^{\text {co. } r \text { r }}(\mathbf{R})$ & $m=1$ or $p=1$ \\
\hline (iv) & $L_{m, n, p}^{\text {co.cr. }}(\mathbf{R})$ & $m=1$ or $p=1$ \\
\hline (v) & $L_{m, n, p}^{p}(\mathbf{R})$ & $m=1$ or $p=1$ or $n=m$ or $n=p$ \\
\hline (vi) & $L_{m, n, p}^{\rho, \lambda}(\mathbf{R})$ & $m=1$ or $p=1$ or $n=m$ or $n=p$ \\
\hline
\end{tabular}

(i) If $L^{\prime}$ is a $G L_{n}(\mathbf{R})$ invariant subspace of $L_{m, n . p}(\mathbf{R})$ such that $L_{m, n, p}^{c o, c r, \lambda}(\mathbf{R}) \subseteq L^{\prime} \subseteq L_{m, n, p}^{c o, c r}(\mathbf{R})$ then there exists a canonical form on $L^{\prime}$ iff $m=1$ or $p=1$.

(ii) If $L^{\prime}$ is a $G L^{n}(\mathbf{R})$ invariant subspace of $L_{m, n, p}(\mathbf{R})$ such that $L_{m, n, p}^{\rho, \lambda}(\mathbf{R}) \subseteq L^{\prime} \subseteq L_{m, n, p}^{\rho}(\mathbf{R})$ then there exists a cont inuous canonical form on $L^{\prime}$ iff $m=1$ or $p=1$ or $n=m$ or $n=p$.

There are many more theorems of this kind. The first step in proving such a theorem is to examine the orbit spaces $L^{\prime} / G L_{n}(\mathbf{R})$. If these "quotient" spaces are not Hausdorff, continuous canonical forms cannot exist. (Jump phenomena again). Section 2 below is mainly concerned with the structure of $L_{m, n, p}^{c r}(\mathbf{R}) / G L_{n}(\mathbf{R})$ and the fibre bundle $L_{m, n, p}^{c r}(\mathbf{R}) \rightarrow L_{m, n, p}^{c r}(\mathbf{R}) / G L_{n}(\mathbf{R})$. In section 3 we then use these results and some examples to prove the theorem 1.7 quoted above.

1.8. Thus theorem 1.7 shows that as a rule one cannot expect continuous canonical forms to exist. However, the next best thing does exist: the quotient space $M_{m . n . p}^{c r}(\mathbf{R})=L_{m . n . p}^{c r}(\mathbf{R}) / G L_{n}(\mathbf{R})$ admits a fine moduli space structure which very roughly means that it is possible to define a "family of linear dynamical systems" over $M_{m . n . p}^{c r}(\mathbf{R})$ such that up to $G L_{n}(\mathbf{R})$-equivalence every completely reachable system occurs exactly once in this family and such that every family can 
be obtained from this (universal) one in precisely one way (by pullback). Section 4 below is concerned with these notions.

1.9 There also exists an algebraic geometric version of the theory presented in this paper. In fact practically all the essential constructions are algebraic with the notable exception of the example 3.3. In the algebraic geometric case this example has to be replaced by a three dimensional one (a one dimensional one does not exist). For this algebraic geometry theory cf. [5] and also [1], [2] and [6], where rather more powerful algebraic-geometric machinery is thrown at the problem.

1.10. The contents of the paper are

1. Introduction and statement of some of the results

2. The quotient manifold $M_{m . n . p}^{\text {cr }}(\mathbf{R})$

2.1. The quotient space $M_{m, n, p}(\mathbf{R})$

2.2. Completely reachable and completely observable systems

2.3. Nice selections

2.4. The local quotients $U_{\alpha} / G L_{n}(\mathbf{R})$

2.5. The differentiable manifolds $M_{m, n, p}^{c r}(\mathbf{R})$ and $M_{m, n, p}^{c r . c o}(\mathbf{R})$

2.6. The principal fibre bundle $L_{m, n, p}^{c r}(\mathbf{R}) \rightarrow M_{m, n, p}^{c r}(\mathbf{R})$

2.7. Remark on the local canonical forms $c_{\# \alpha}$

3. Existence and nonexistence of continuous canonical forms

3.1. Local canonical forms and local sections of $L_{m, n, p}^{c r}(\mathbf{R}) \rightarrow M_{m, n, p}^{c r}(\mathbf{R})$

3.2. Examples

3.3. An embedding $\mathbf{P}^{1}(\mathbf{R}) \rightarrow M_{m, n, p}^{c r, c o}(\mathbf{R})$

3.4. Some remarks on principal $G L_{n}(\mathbf{R})$ bundles

3.5. On the nonexistence of continuous canonical forms

4. Families of linear dynamical systems and the fine moduli space $M_{m, n . p}^{c r}(\mathbf{R})$.

4.1. Families of linear dynamical systems

4.2. Description of families of linear dynamical systems by transition functions

4.3. The universal family $\Sigma^{u}$ over $M_{m, n, p}^{c r}(\mathbf{R})$

4.4. The functor $\Phi_{m . n . p}$ of isomorphism classes of linear dynamical systems.

4.5. The fine moduli space $M_{m, n, p}(\mathbf{R})$

4.6. Remarks.

\section{The Quotient Manifold $M_{m, n, p}^{c r}(\mathbf{R})$}

In this section we study the action of $G L_{n}(\mathbf{R})$ on $L_{m . n . p}(\mathbf{R})$ and discuss the quotient spaces $L^{\prime} / G L_{n}(\mathbf{R})$ for various $G L_{n}(\mathbf{R})$-invariant subspaces $L^{\prime}$.

2.1. The Quotient Space $M_{m, n, p}(\mathbf{R})$. We define $M_{m, n, p}(\mathbf{R})$ as the quotient space of $L_{m, n, p}(\mathbf{R})$ under $G L_{n}(\mathbf{R})$ equivalence as defined in 1.4. I.e. the points of $M_{m, n, p}(\mathbf{R})$ are the orbits of $G L_{n}(\mathbf{R})$ in $L_{m, n, p}(\mathbf{R})$ and its topology is the finest topology for which the natural projection $\pi: L_{m, n, p}(\mathbf{R}) \rightarrow M_{m, n, p}(\mathbf{R})$ is continuous. The space $M_{m, n, p}(\mathbf{R})$ is never a Hausdorff space. (Because of the Jump phenomena mentioned in the introduction above; or in other words, because not all orbits of $G L_{n}(\mathbf{R})$ in $L_{m, n, p}(\mathbf{R})$ are closed subsets).

2.2 Completely Reachable and Completely Observable Systems. Let $(F, G$. 
$H) \in L_{m, n, p}(\mathbf{R})$. The system $(F, G, H)$ is said to be completely reachable if the matrix $R(F, G)$ has rank $n$, where $(R(F, G)$ is the matrix

$$
R(F, G)=\left(G F G \ldots F^{n} G\right)
$$

consisting of the columnvectors $F^{i} G_{j}, i=0, \ldots, n ; j=1, \ldots, m$, where $G_{j}, j$ $=1, \ldots, m$ is the $j$-th column of $G$. Dually the system $(F, G, H)$ is said to be completely observable if the matrix $Q(F, H)$ has rank $n$, where $Q(F, H)$ is the matrix defined by

$$
Q(F, H)^{\prime}=\left(H^{\prime} F^{\prime} H^{\prime} \ldots F^{\prime n} H^{\prime}\right)
$$

where $A^{\prime}$ denotes the transposed matrix of $A$. Let

$$
\begin{aligned}
& L_{m, n, p}^{c r}(\mathbf{R})=\left\{(F, G, H) \in L_{m, n, p}(\mathbf{R}) \mid \operatorname{rank}(R(F, G))=n\right\} \\
& L_{m, n, p}^{c o}(\mathbf{R})=\left\{(F, G, H) \in L_{m, n, p}(\mathbf{R}) \mid \operatorname{rank}(Q(F, H))=n\right\} \\
& L_{m, n, p}^{c r, c o}(\mathbf{R})=L_{m, n, p}^{c r}(\mathbf{R}) \cap L_{m, n, p}^{c o}(\mathbf{R})
\end{aligned}
$$

We define

$$
M_{m, n, p}^{w}(\mathbf{R})=\pi\left(L_{m, n, p}^{w}(\mathbf{R})\right)
$$

where $w$ stands for $c r$ or co or $c r, c o$.

2.3. Nice Selections. We number the $m(n+1)$ columns of $R(F, G)$ by pairs of indices as follows

$$
01, \ldots, 0 m ; 11, \ldots, 1 m ; \ldots ; n 1, \ldots, n m
$$

and use $J_{n, m}$ to denote this ordered set. A nice selection $\alpha$ is now defined as an ordered subset $\alpha \in J_{n, m}$ of size $n$ such that $(i, j) \in \alpha$ implies $\left(i^{\prime}, j\right) \in \alpha$ for all $i^{\prime} \leq i$. If $\alpha$ is a nice selection we define $s(\alpha, j), j=1, \ldots, m$ as that element $(k, j) \in J_{n, m}$ such that $\left(k^{\prime}, j\right) \in \alpha$ for all $0 \leq k^{\prime}<k$. (If $\alpha$ contains no elements of the form $(i, j)$ then $s(\alpha$, $j)=(0, j))$. These $s(\alpha, j)$ are called the successor indices of $\alpha$. There are precisely $m$ of them; one for each $j=1, \ldots, m$. If $\alpha$ is any subset of $J_{n, m}$ we denote with $R(F$, $G)_{\alpha}$ the matrix obtained from $R(F, G)$ by removing all columns whose index is not in $\alpha$.

2.3.1. LEMMA.If $(F, G, H) \in L_{m, n, p}^{c r}(\mathbf{R})$ then there is a nice selection $\alpha$ such that $\operatorname{det}\left(R(F, G)_{\alpha}\right) \neq 0$.

Proof. Cf. [1] lemma 2.4.1.

2.4. The Local Quotients $U_{\alpha} / G L_{n}(\mathbf{R})$.

Let $\alpha$ be a nice selection. We define

$$
\begin{aligned}
& U_{\alpha}=\left\{(F, G, H) \in L_{m, n, p}(\mathbf{R}) \mid \operatorname{det} R(F, G)_{\alpha} \neq 0\right\} \\
& W_{\alpha}=\left\{(F, G, H) \in L_{m, n, p}(\mathbf{R}) \mid R(F, G)_{\alpha}=I_{n}\right\}
\end{aligned}
$$

where $I_{n}$ is the $n \times n$ identity matrix. We claim that $W_{\alpha}$ is naturally homeomorphic with $\mathbf{R}^{m n+n p}$. To see this write $x \in \mathbf{R}^{m n}$ as a sequence of $m$ column vectors of length $n$ as follows $x=\left(x_{1}, \ldots, x_{m}\right)$ where $x_{1}$ consists of the first $n$ coordinates of $x, x_{2}$ of the second $n$ coordinates of $x$, etc. 
2.4.3. LEMMA.For each $x \in \mathbf{R}^{m n}$ there is precisely one pair of matrices $(F, G)$ of sizes $n \times n$ and $n \times m$ respectively such that

$$
R(F, G)_{\alpha}=I_{n}, R(F, G)_{s(\alpha, j)}=x_{j}, j=1, \ldots, m
$$

Proof. Cf. [1] lemma 2.3.3.

2.4.5. Now let $x=(y, z) \in \mathbf{R}^{m n} \times \mathbf{R}^{n p}$; we define $\psi_{\alpha}(x)=\left(F_{\alpha}(x), G_{\alpha}(x), H_{\alpha}(x)\right)$ as the unique triple of matrices such that $\left(F_{\alpha}(x), G_{\alpha}(x)\right)$ is the unique pair corresponding to $y \in \mathbf{R}^{m n}$ as in lemma 2.4 .3 and such that $H_{\alpha}(x)$ is the $p \times n$ matrix corresponding to $z$. Lemma 2.4 .3 now implies that $\psi_{\alpha}$ is a homeomorphism $\mathbf{R}^{m n}$ $\times \mathbf{R}^{n p} \rightarrow W_{\alpha}$.

2.4.6. Let $X$ be any topological space. We let $G L_{n}(\mathbf{R})$ act on $G L_{n}(\mathbf{R}) \times X$ by multiplication on the left hand factor.

There now is a natural $G L_{n}(\mathbf{R})$-invariant morphism $t_{\alpha}$ defined as follows

$$
\begin{gathered}
t_{\alpha}: U_{\alpha} \rightarrow G L_{n}(\mathbf{R}) \times W_{\alpha}, \\
(F, G, H) \mapsto\left(T^{-1},(F, G, H)^{T}\right), \text { where } T=R(F, G)_{\alpha}^{-1} .
\end{gathered}
$$

2.4.8. LEMMA. $t_{\alpha}$ is a $G L_{n}(\mathbf{R})$-invariant homeomorphism.

Proof. One obviously has for all $\bar{T} \in G L_{n}(\mathbf{R})$,

$$
R\left(\bar{T} F \bar{T}^{-1}, \bar{T} G\right)=\bar{T} R(F, G) .
$$

The $G L_{n}(\mathbf{R})$-invariance of $t_{\alpha}$ follows immediately from this. To see that $t_{\alpha}$ is a homeomorphism observe that $(T,(F, G, H)) \mapsto(F, G, H)^{T}$ is an inverse map to $t_{x^{-}}$

2.5. The Differentiable Manifolds $M_{m . n . p}^{c r}(\mathbf{R})$ and $M_{m . n . p}^{c r, c o}(\mathbf{R})$. By means of the results of 2.4 we can now obtain a local pieces and patching data description of the topological space $M_{m . n . p}^{c r}(\mathbf{R})$. We see from 2.4 .8 and 2.4 .5 that $U_{\alpha} / G L_{n}(\mathbf{R})$ $\simeq \mathbf{R}^{m n+n p}$. It remains to patch these local pieces together.

We define for each pair of nice selections $\alpha, \beta$

$$
\begin{aligned}
V_{\alpha} & =\mathbf{R}^{m n+n p}, V_{\alpha}^{c o}=\left\{x \in V_{\alpha} \mid \operatorname{rank} Q\left(F_{\alpha}(x), H_{\alpha}(x)\right)=n\right\} \\
V_{\alpha \beta} & =\left\{x \in V_{\alpha} \mid \operatorname{det}\left(R\left(F_{\alpha}(x), G_{\alpha}(x)\right)_{\beta}\right) \neq 0\right\} \\
V_{\alpha \beta}^{c o} & =V_{\alpha \beta} \cap V_{\alpha}^{c o}
\end{aligned}
$$

We now define homeomorphisms $\phi_{\alpha \beta}: V_{\alpha \beta} \rightarrow V_{\beta \alpha}$ as follows

$$
\begin{aligned}
& \phi_{\alpha \beta}(x)=y \Leftrightarrow\left(F_{\alpha}(x), G_{\alpha}(x), H_{\alpha}(x)\right)^{T}=\left(F_{\beta}(y), G_{\beta}(y), H_{\beta}(y)\right) \\
& \text { with } T=R\left(F_{\alpha}(x), G_{\alpha}(x)\right)_{\beta}^{-1} .
\end{aligned}
$$

One easily checks that for all triples of nice selections $\alpha, \beta, \gamma, \phi_{\beta \gamma} \phi_{\alpha \beta}(x)=\phi_{x_{\gamma}}(x)$ whenever the left hand side is defined and that $\phi_{\alpha \beta}$ induces homeomorphisms $\phi_{\alpha \beta}^{c o}$ : $V_{\alpha \beta}^{c o} \rightarrow V_{\beta \alpha}^{c o}$.

2.5.5. LEMMA. The topological space obtained by glueing together the $V_{\alpha} b y$ ' means of the $\phi_{\alpha \beta}$ is $M_{m, n, p}^{c r}(\mathbf{R})$. More precisely we have

(i) $\quad V_{\alpha} \stackrel{\psi_{\alpha}}{\rightarrow} W_{\alpha} \subset U_{\alpha} \subset L_{m, n, p}^{c r}(\mathbf{R}) \rightarrow M_{m, n, p}^{c r}(\mathbf{R})$ is injective. Let $\psi_{\alpha}^{\prime}$ be this composite map. Then

(ii) $\psi_{\alpha}^{\prime}(x)=\psi_{\beta}^{\prime}(y)$ if and only if $x \in V_{\alpha \beta}$ and $\phi_{\alpha \beta}(x)=y$.

(iii) $M_{m, n, p}^{c r}(\mathbf{R})=\cup_{\alpha} \psi_{\alpha}^{\prime}\left(V_{\alpha}\right)$ where $\alpha$ runs through all nice selections. 
Proof. (i): If $(F, G, H),(\bar{F}, \bar{G}, \bar{H}) \in W_{x}$ are $G L_{n}(\mathbf{R})$ equivalent. Then, cf. (2.4.9) we must have $R(\bar{F}, \bar{G})=T R(F, G)$ for some $T \in G L_{n}(\mathbf{R})$. Hence $R(\bar{F}, \bar{G})_{\alpha}=T R(F, G)_{\alpha}$. But $R(F, G)_{\alpha}=R(\bar{F}, \bar{G})_{\alpha}=I_{n}$ : hence $T=I_{n}$. Part (ii) of the lemma follows directly from the definition of $\phi_{x \beta}$ (cf. (2.5.4)), and part (iii) follows from lemma 2.3.1 and 2.4.8.

\subsubsection{COROLLARY.}

(i) $M_{m, n, p}^{c r, c o}(\mathbf{R})$ is the topological space obtained by glueing together the $V_{x}^{c o} b y$ means of the induced homeomorphisms $\phi_{\alpha \beta}^{c o}: V_{\alpha \beta}^{c o} \rightarrow V_{\beta \alpha}^{c o}$.

(ii) The $\psi_{\alpha}^{\prime}\left(V_{\alpha}\right)$ and $\psi_{\alpha}^{\prime}\left(V_{\alpha}^{c o}\right)$ are open subsets of $M_{m, n, p}^{c r}(\mathbf{R})$ and $M_{m, n, p}^{c r, c o}(\mathbf{R})$ respectively.

We now want to show that $M_{m, n, p}^{c r}(\mathbf{R})$ is a Hausdorff space. To do this we use the following lemma.

2.5.7. LEMMA. Let $\alpha, \beta$ be two nice selections and suppose that $\left(G_{i}, G_{i}, H_{i}\right), i \in \mathbf{N}$ is a sequence of elements in $U_{x}$ converging to $(F, G, H) \in U_{\alpha}$ as $i \rightarrow \infty$ and that $\left(\bar{F}_{i}, \bar{G}_{i}\right.$, $\left.\bar{H}_{i}\right), i \in \mathbf{N}$ is a sequence of elements in $U_{\beta}$ converging to $(\bar{F}, \bar{G}, \bar{H}) \in U_{\beta}$. Suppose moreover that $\left(F_{i}, G_{i}, H_{i}\right)$ and $\left(\bar{F}_{i}, \bar{G}_{i}, \bar{H}_{i}\right)$ are $G L_{n}(\mathbf{R})$ equivalent for all $i \in \mathbf{N}$. Then $(F$, $G, H)$ and $(\bar{F}, \bar{G}, \bar{H})$ are $G L_{n}(\mathbf{R})$-equivalent.

Proof. We have

$$
\lim _{i \rightarrow \infty} R\left(F_{i}, G_{i}\right)_{\alpha}=R(F, G)_{\alpha}
$$

Now $R\left(F_{i}, G_{i}\right)_{\alpha}$ and $R(F, G)_{\alpha}$ are invertible for all $i \in \mathbf{N}$. This means that also

$$
\lim _{i \rightarrow \infty} R\left(F_{i}, G_{i}\right)_{\alpha}^{-1}=R(F, G)_{\alpha}^{-1}
$$

We also have that

$$
\lim _{i \rightarrow \infty} R\left(\bar{F}, \bar{G}_{i}\right)_{\alpha}=R(\bar{F}, \bar{G})_{\alpha}
$$

Now because $\left(F_{i}, G_{i}, H_{i}\right)$ and $\left(\bar{F}_{i}, \bar{G}_{i}, \bar{H}_{i}\right)$ are both in $L_{m, n, p}^{c r}(\mathbf{R})$ they are $G L_{n}(\mathbf{R})$ equivalent if and only if

$$
\text { (2.5.11) } \quad\left(F_{i}, G_{i}, H_{i}\right)^{T_{i}}=\left(\bar{F}_{i}, \bar{G}_{i}, \bar{H}_{i}\right) \text { with } T_{i}=R\left(\bar{F}_{i}, \bar{G}_{i}\right)_{\alpha} R\left(F_{i}, G_{i}\right)_{\alpha}^{-1}
$$

This follows from the fact that $T_{i}$ must be such that $T_{i} R\left(F_{i}, G_{i}\right)=R\left(\bar{F}_{i}, \bar{G}_{i}\right)$ and that $R\left(F_{i}, G_{i}\right)$ and $R\left(\bar{F}_{i}, \bar{G}_{i}\right)$ both have rank $n$. By (2.5.9) and (2.5.10) we know that $\lim _{i \rightarrow \infty} T_{i}$ exists and is equal to $R(F, G)_{\alpha} R(\bar{F}, \bar{G})_{\alpha}^{-1}=T_{\infty}$ and taking the limit for $i$ $\rightarrow \infty$ in the equality $T_{i} R\left(F_{i}, G_{i}\right)=R\left(\bar{F}_{i}, \bar{G}_{i}\right)$ we find

$$
T_{\propto} R(F, G)=R(\bar{F}, \bar{G})
$$

Both $R(F, G)$ and $R(\bar{F}, \bar{G})$ are of rank $n$ so that rank $\left(T_{\infty}\right)=n$, i.e. $T_{\infty}$ is invertible so that

$$
\lim _{i \rightarrow \infty} T_{i}^{-1}=T_{\infty}^{-1}
$$

We already had

$$
\lim _{i \rightarrow \infty} T_{i}=T_{\propto}
$$


and

$$
\bar{F}_{i}=T_{i} F_{i} T_{i}^{-1}, \bar{G}_{i}=T_{i} G_{i}, \bar{H}_{i}=H_{i} T_{i}^{-1}
$$

Taking the limit for $i \rightarrow \infty$ of the equalities (2.5.15) now shows that

$$
(\bar{F}, \bar{G}, \bar{H})=(F, G, H)^{T_{\infty}}
$$

which proves the lemma.

2.5.17. THEOREM. $M_{m, n, p}^{c r}(\mathbf{R})$ is a differentiable manifold and $M_{m, n, p}^{c r, c o}(\mathbf{R})$ is a differentiable submanifold.

Proof. The patching functions $\phi_{\alpha \beta}$ and $\phi_{\alpha \beta}^{c o}$ are $n$ times differentiable for all $n$. In view of lemmas 2.5.5 and 2.5.6 it therefore suffices to prove that $M_{m, n, p}^{\text {cr }}(\mathbf{R})$ is a Hausdorff space. This follows from the fact that $M_{m, n, p}^{c r}(\mathbf{R})$ is covered by the Hausdorff open subsets $V_{x}^{\prime} \simeq \mathbf{R}^{m n+n p}$ and lemma 2.5.7 above which says that a sequence in $V_{\alpha} \cap V_{\beta}$ cannot converge to two different points (one in $V_{\alpha}$ and one in $V_{\beta}$ ) at the same time. (If $M_{m, n, p}^{c r}(\mathbf{R})$ were not Hausdorff such a sequence would exist).

2.5.18. Remark. The manifold $M_{m, n, p}^{c r}(\mathbf{R})$ is never a compact manifold.

2.6.The principal fibre bundle. $L_{m, n, p}^{c r}(\mathbf{R}) \stackrel{\pi}{\rightarrow} M_{m, n, p}^{c r}(\mathbf{R})$.

From now on we shall occasionally talk about fibre bundles and principal fibre bundles over a topological space $X$. For these concepts and some elementary facts concerning them the reader is referred to [4]. All fibre bundles in this paper will be locally trivial and we shall often omit to mention this.

According to lemmas 2.5 .5 and 2.4.8 we have a commutative diagram

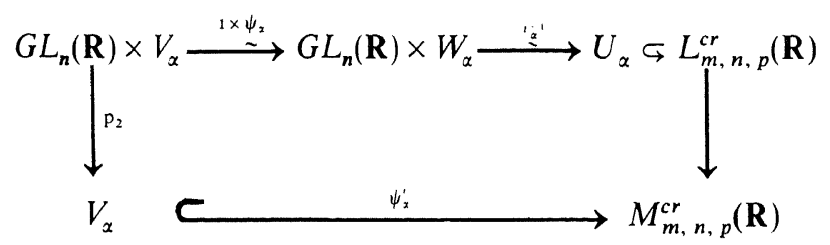

where $p_{2}$ is the projection onto the second factor. By lemma 2.5 .5 and corollary 2.5.6 we know that the $V_{\alpha}^{\prime}=\psi_{\alpha}^{\prime}\left(V_{\alpha}\right)$ are open and form an open covering of $M_{m, n, p}^{c r}(\mathbf{R})$ where $\alpha$ runs through all nice selections.

We now obtain from (2.6.1) a commutative diagram

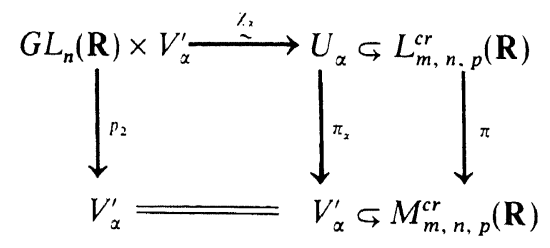

where $\pi_{\alpha}$ is the restriction of $\pi$ to $U_{\alpha}$ and $\chi_{\alpha}$ is equal to

$$
\left.\chi_{x}=t_{\alpha}^{-1}\left(1 \times \psi_{\alpha}\right)\left(1 \times \psi_{x}^{\prime}\right)^{-1} t \times 1\right)
$$

where $l: G L_{n}(\mathbf{R}) \rightarrow G L_{-}(\mathbf{R})$ is the homeomorphism $T \mapsto T^{-1}$. 
The homeomorphism $\chi_{\alpha}$ can be described as follows. Let $x \in V_{\alpha}^{\prime} \subset M_{m, n, p}^{c r}(\mathbf{R})$, let $(F, G, H) \in \pi^{-1}(x)$. Then we have

$$
\begin{aligned}
& \chi_{\alpha}\left(I_{n}, x\right)=(F, G, H)^{T} \text { with } T=R(F, G)_{\alpha}^{-1} \\
& \chi_{\alpha}(\bar{T}, x)=(F, G, H)^{T \bar{T}}
\end{aligned}
$$

Now let $(T, x) \in G L_{n}(\mathbf{R}) \times\left(V_{\alpha}^{\prime} \cap V_{\beta}^{\prime}\right)$. Then we know (by the commutativity of diagram (2.6.2) above) that $\chi_{\beta}^{-1} \chi_{\alpha}$ is well defined and of the form

$$
\chi_{\beta}^{-1} \chi_{\alpha}(T, x)=\left(\rho_{\alpha \beta}(x, T), x\right)
$$

We are going to calculate $\rho_{\alpha \beta}(x, T)$.

Let $x_{\alpha}^{\prime}=\psi_{x}^{\prime-1}(x)$. Then we have

$$
\begin{aligned}
\left(\rho_{\alpha \beta}(x, T), x\right)= & (l \times 1)^{-1}\left(1 \times \psi_{\beta}^{\prime}\right)\left(1 \times \psi_{\beta}\right)^{-1} t_{\beta} t_{\alpha}^{-1}\left(1 \times \psi_{\alpha}\right)\left(1 \times \psi_{\alpha}^{\prime}\right)^{-1}(l \times 1) \\
& (T, x) \\
= & (l \times 1)^{-1}\left(1 \times \psi_{\beta}^{\prime}\right)\left(1 \times \psi_{\beta}\right)^{-1} t_{\beta} t_{\alpha}^{-1}\left(1 \times \psi_{\alpha}\right)\left(T^{-1}, x_{\alpha}^{\prime}\right) \\
= & (l \times 1)^{-1}\left(1 \times \psi_{\beta}^{\prime}\right)\left(1 \times \psi_{\beta}\right)^{-1} t_{\beta} t_{\alpha}^{-1}\left(T^{-1},\right. \\
& \left(F_{\alpha}\left(x_{\alpha}^{\prime}\right), G_{\alpha}\left(x_{\alpha}^{\prime}\right), H_{\alpha}\left(x_{\alpha}^{\prime}\right)\right) \\
= & (l \times 1)^{-1}\left(1 \times \psi_{\beta}^{\prime}\right)\left(1 \times \psi_{\beta}\right)^{-1} t_{\beta}\left(\left(F_{\alpha}\left(x_{\alpha}^{\prime}\right), G_{\alpha}\left(x_{\alpha}^{\prime}\right), H_{\alpha}\left(x_{\alpha}^{\prime}\right)\right)^{T-1}\right.
\end{aligned}
$$

This shows that (cf. the definition of $t_{\beta}(2.4 .7)$ )

$$
\rho_{x \beta}(x, T)=R\left(T^{-1} F_{\alpha}\left(x_{\alpha}^{\prime}\right) T, T^{-1} G_{\alpha}\left(x_{\alpha}^{\prime}\right)\right)_{\beta}^{-1}
$$

I.e.

$$
\rho_{\alpha \beta}(x, T)=R\left(F_{\alpha}\left(\psi_{\alpha}^{\prime-1}(x)\right), G_{\alpha}\left(\psi_{\alpha}^{\prime-1}(x)\right)\right)_{\beta}^{-1} T
$$

We have now proved:

2.6.6 THEOREM. $L_{m, n, p}^{c r}(\mathbf{R}) \rightarrow M_{m, n, p}^{c r}(\mathbf{R})$ is a principal (locally trivial) fibre bundle with group $G L_{n}(\mathbf{R})$.

Proof. This follows from 2.5.6, the commutativity of (2.6.2) and (2.6.5); cf. Steenrod [4], 2.3 and 8.1.

2.6.7. COROLLARY. The covering $\left\{V_{\alpha}^{\prime}\right\}$ of $M_{m, n, p}^{c r}(\mathbf{R})$ and the transition functions defined by (2.6.5) define the principal fibre bundle $L_{m, n, p}^{c r}(\mathbf{R})$ $\rightarrow M_{m, n, p}^{c r}(\mathbf{R})$.

2.6.8. COROLLARY. $L_{m, n, p}^{\text {cr.co }}(\mathbf{R}) \rightarrow M_{m, n, p}^{\text {cr.co }}(\mathbf{R})$ is a (locally trivial) principal fibre bundle with group $G L_{n}(\mathbf{R})$. It is the restriction of the bundle $L_{m, n, p}^{c r}(\mathbf{R})$ $\rightarrow M_{m, n, p}^{c r}(\mathbf{R})$ to the subspace $M_{m, n, p}^{c r, c o}(\mathbf{R})$.

2.7. Remark on the local canonical forms $c_{\# \alpha^{*}}$ The constructions and calculations carried out in the sections above are very much related to certain (currently popular) local continuous canonical forms

$$
c_{\# x}: U_{x} \rightarrow U_{x}
$$

where $\alpha$ is a nice selection. These are defined as follows

(2.7.2) $c_{\#}(\alpha)(F, G, H)=\left(R(F, G)_{\alpha}^{-1} F R(F, G)_{\alpha}, R(F, G)_{\alpha}^{-1} G, H R(F, G)_{\alpha}^{-1}\right)$ 
The relation between the $c_{\# \alpha}$ and the various maps above is as follows (cf. (2.6.4)).

$$
c_{\# \alpha}((F, G, H))=\chi_{\alpha}\left(I_{n}, \pi_{\alpha}(F, G, H)\right)
$$

Cf. also diagram (2.6.2). If we define

$$
s_{\alpha}: V_{\alpha}^{\prime} \rightarrow U_{\alpha}, x \mapsto \chi_{\alpha}\left(I_{n}, x\right)
$$

then we have

$$
\pi_{\alpha} \mathrm{s}_{\alpha}=1_{V_{\dot{\alpha}}}
$$

i.e. $s_{\alpha}$ is a local section of the bundle $L_{m, n, p}^{c r}(\mathbf{R}) \rightarrow M_{m, n, p}^{c r}(\mathbf{R})$ and the canonical forms $c_{\# x}$ are related to these sections by

$$
c_{\# \alpha}=s_{\alpha} \pi_{\alpha} \text {. }
$$

\section{Existence and Nonexistence of Continuous Canonical Forms}

We are now in a position to start investigating whether continuous canonical forms exist or not.

3.1 Local Canonical Forms and Local Sections of $L_{m, n, p}^{c r}(\mathbf{R}) \stackrel{\pi}{\rightarrow} M_{m, n, p}^{c r}(\mathbf{R})$. Let $L^{\prime} \subset L_{m, n, p}^{c r}(\mathbf{R})$ be a $G L_{n}$-invariant subset of $L_{m, n, p}^{c r}(\mathbf{R})$ and let $M^{\prime}=\pi\left(L^{\prime}\right)$. Then we have an (induced) principal $G L_{n}(\mathbf{R})$ bundle $L^{\prime} \stackrel{{ }^{\prime}}{\rightarrow} M^{\prime}$.

3.1.1. LEMMA. Let $\phi: L^{\prime} \rightarrow Y$ be any cont inuous map such that $\phi$ is constant on the orbits of $G L_{n}(\mathbf{R})$ in $L^{\prime}$. Then there is a unique map $\psi: M^{\prime} \rightarrow Y$ such that $\phi=\psi \pi^{\prime}$.

Proof. Let $U_{\alpha}^{\prime}=L^{\prime} \cap U_{\alpha}, V_{\alpha}^{\prime \prime}=V_{\alpha}^{\prime} \cap M^{\prime}$. Then we have a commutative diagram

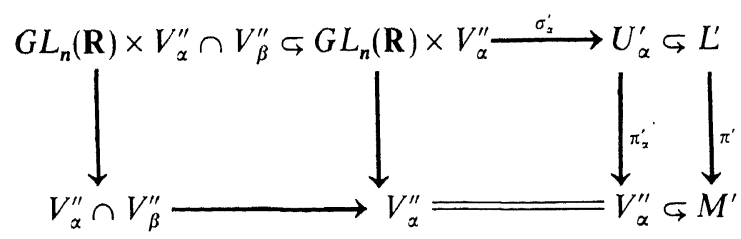

where $\sigma_{\alpha}^{\prime}$ is a $G L_{n}(\mathbf{R})$-invariant homeomorphism, viz. the restriction to $G L_{n}(\mathbf{R})$ $\times V_{\alpha}^{\prime \prime}$ of $t_{\alpha}^{-1}\left(1 \times \psi_{\alpha}\right)\left(1 \times \psi_{\alpha}^{\prime}\right)^{-1}$. Let $\phi_{\alpha}$ be the restriction of $\phi$ to $U_{\alpha}^{\prime}$. Then the middle commutative square of 3.1 .2 shows that there is a unique $\psi_{\alpha}: V_{\alpha}^{\prime \prime} \rightarrow Y$ such that $\psi_{\alpha} \pi_{\alpha}^{\prime}=\phi_{\alpha}$. In fact we have $\psi_{\alpha}(x)=\phi_{\alpha}{ }_{\alpha}^{t_{\alpha}^{-1}}\left(I_{n}, x\right)$. It follows that $\psi_{\alpha} \mid \mathrm{V}_{\alpha}^{\prime \prime} \cap V_{\beta}^{\prime \prime}$ $=\psi_{\beta} \mid V_{\alpha}^{\prime \prime} \cap V_{\beta}^{\prime \prime}$, so that the $\psi_{\alpha}$ combine to define a continuous map $\psi: M^{\prime} \rightarrow Y$ such that $\psi \pi^{\prime}=\phi$. The map $\psi$ is unique because we must have $\psi \mid V_{\alpha}^{\prime \prime}=\psi_{\alpha}$ for all nice selections $\alpha$.

3.1.2 LEMMA. There exists a continuous form $c^{\prime}$ on $L^{\prime}$ if and only if there is a section $s^{\prime}$ of the bundle $\pi^{\prime}: \mathrm{L}^{\prime} \rightarrow \mathrm{M}^{\prime}$, i.e., a continuous map $\mathrm{s}^{\prime}: M^{\prime} \rightarrow L^{\prime}$ such that $\pi^{\prime} s^{\prime}$ $=1_{M}$.

Proof. Suppose $s^{\prime}: M^{\prime} \rightarrow L^{\prime}$ is a section. Then $s^{\prime} \pi^{\prime}: L^{\prime} \rightarrow L^{\prime}$ is a continuous canonical form on $L^{\prime}$. This follows immediately because $\pi^{\prime} s^{\prime}=1_{M}$, and because $\pi^{\prime}$ induces a one-to-one onto correspondence between the orbits of $G L_{n}(\mathbf{R})$ in $L^{\prime}$ and 
the points of $M^{\prime}$. Inversely suppose that $c^{\prime}: L^{\prime} \rightarrow L^{\prime}$ is a continuous canonical form. Then $c^{\prime}$ is constant on the orbits of $G L_{n}(\mathbf{R})$ in $L^{\prime}$, therefore by lemma 3.1.1 there is an induced map $s^{\prime}: M^{\prime} \rightarrow L^{\prime}$ such that $s^{\prime} \pi^{\prime}=c^{\prime}$. One checks easily that $s^{\prime}$ is indeed a section.

3.1.3. Remark. The induced principal $G L_{n}(\mathbf{R})$ bundle $L^{\prime} \rightarrow M^{\prime}$ admits a section if and only if it is (isomorphic to) the trivial principal $G L_{n}(\mathbf{R})$-bundle over $M^{\prime}$. Cf. Steenrod [4], 8.3. Thus there are canonical forms on each of the $U_{\alpha} \subset L_{m, n, p}^{c r}(\mathbf{R}), \alpha$ a nice selection. $\mathrm{Cf}$. also section 2.7 above.

\subsubsection{COROLLARY.}

(i) If $m=1$ there is a continuous canonical form on $L_{m, n, p}^{c r}(\mathbf{R})$

(ii) If $m=n$ there is a continuous canonical form on $L_{m, n, p}^{\rho}(\mathbf{R})$. Proof.

(i) If $m=1$ there is only one nice selection viz. $\alpha=\{(0,1),(1,1), \ldots,(n-1), 1)\}$. So that in this case $L_{m, n, p}^{c r}(\mathbf{R})=\mathrm{U}_{\alpha}$. And we know that the induced bundle $U_{\alpha} \rightarrow V_{\alpha}^{\prime}=\pi\left(U_{\alpha}\right)$ has a section, e.g. $s_{\alpha}: V_{\alpha}^{\prime} \rightarrow U_{\alpha}, x \mapsto t_{\alpha}^{-1}\left(I_{n}, x\right)$.

(ii) By definition $(F, G, H) \in L_{m, n, p}^{\rho}(\mathbf{R})$ implies that $\operatorname{rank} G=\min (m, n)$. So if $m$ $=n$ we have $\operatorname{rank}(G)=n$, which says that $L_{m, n, p}^{\rho}(\mathbf{R})$ in this case is contained in $U_{\beta}$ where $\beta$ is the nice selection $\beta=\{(0,1),(0,2), \ldots,(0, \mathrm{n})\}$.

3.1.5. Duality. The assignment

$$
\delta:(F, G, H) \mapsto\left(F^{\prime}, H^{\prime}, G^{\prime}\right)
$$

defines a homeomorphism $L_{m, n, p}(\mathbf{R}) \rightarrow L_{p, n, m}(\mathbf{R})$. The map $\delta$ is not $G L_{n}(\mathbf{R})-$ invariant but it does have the property that two triples $(F, G, H),(\bar{F}, \bar{G}, \bar{H}) \in L_{m, n, p}^{c r}$ are $G L_{n}(\mathbf{R})$ equivalent if and only if the triples $\delta(F, G, H)$ and $\delta(\bar{F}, \bar{G}, \bar{H})$ are $G L_{n}(\mathbf{R})$ equivalent. Note also that $\delta^{2}=i d$. The duality $\delta$ halves the work we have to do to prove theorems like 1.7. This is proved by the following lemma.

3.1.6. LEMMA. Let $L^{\prime}$ be a $G L_{n}(\mathbf{R})$-invariant subspace of $L_{m, n, p}(\mathbf{R})$, then $\delta\left(L^{\prime}\right)$ is a $G L_{n}(\mathbf{R})$ invariant subspace of $L_{p, n, m}(\mathbf{R})$ and there is a continuous canonical form on $\delta\left(L^{\prime}\right)$ iff there is a continuous canonical form on $L^{\prime}$.

Proof. The lemma is proved by: if $c: L^{\prime} \rightarrow L^{\prime}$ is a continuous canonical form on $L^{\prime}$, then $\delta c^{\prime} \delta^{-1}$ is a continuous canonical form on $\delta\left(L^{\prime}\right)$, and if $c^{\prime}: \delta\left(L^{\prime}\right) \rightarrow \delta\left(L^{\prime}\right)$ is a continuous canonical form on $\delta\left(L^{\prime}\right)$ then $\delta^{-1} c^{\prime} \delta$ is a continuous canonical form on $L$. These last two statements follow immediately from the definition of continuous canonical form (cf. 1.5) and the remarks made above in 3.1.5.

3.1.7. COROLLARY. If $p=1$ there is a continuous canonical form on $L_{m . n . p}^{c o}(\mathbf{R})$. If $p=n$ there is a continuous canonical form on $L_{m, n, p}^{\rho}(\mathbf{R})$.

Proof. These statements follow from 3.1 .4 and 3.1 .6 because $\delta\left(L_{m, n, p}^{c r}(\mathbf{R})\right)$ $\left.=L_{p, n, m}^{c o}(\mathbf{R})\right)$ and $\delta\left(L_{m, n, p}^{\rho}(\mathbf{R})\right)=L_{p, n, m}^{\rho}(\mathbf{R})$.

3.2. Examples. In this section we construct a number of examples of $G, F$ and $H$ matrices which will be useful in our continuous canonical form investigations.

3.2.1 The Matrices $G_{n, m}(t, s)$. These $n \times m$ matrices are as follows (3.2.2) If $n=1, m \geq 2 \quad G_{1, m}(t, s)=\left(\begin{array}{lll}t & 0 & 0\end{array}\right)$ 
(3.2.3) If $n>2,2<m<n \quad G_{n, m}(t, s)=\left(\begin{array}{cc|cc}t & s & 0 \ldots 0 \\ 1 & 1 & 0 \ldots 0 \\ \hline 2 & 1 & \\ \cdot & . & B \\ \cdot & \cdot & \\ \cdot & . & \end{array}\right)$

where $B$ is an $(n-2) \times(m-2)$ matrix independent of $t, s$ such that the columns of $B$ and the columnvector $(1,1, \ldots, 1)^{\prime}$ span an $(m-1)$-dimensioral subspace of $\mathbf{R}^{n-2}$. Note that such a $B$ exists because $2<m<n$.

(3.2.4) If $n>2, m=2 \quad G_{n, 2}(t, s)=\left(\begin{array}{cc}t & s \\ 1 & 1 \\ 2 & 1 \\ \vdots & \vdots \\ 2 & 1\end{array}\right)$

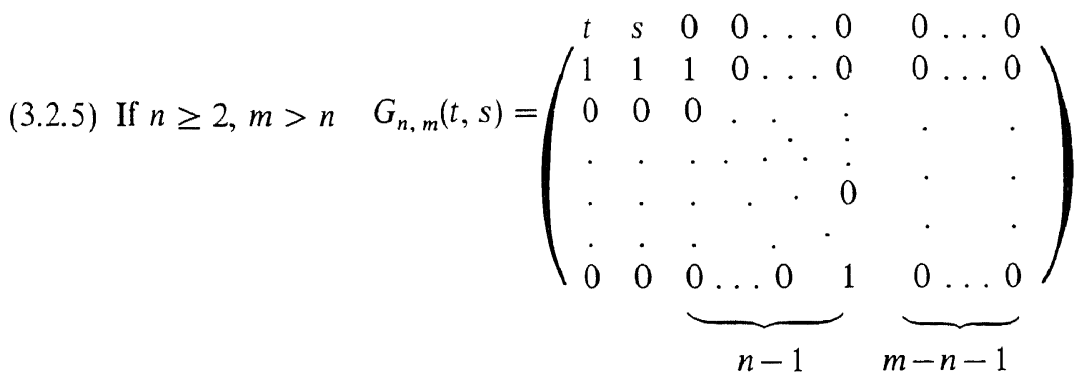

(3.2.6) If $n=m \geq 2 \quad G_{n, n}(t, s)=\left(\begin{array}{ccccc}t & s & 0 & \ldots & 0 \\ 1 & 1 & \cdot & . \\ 2 & 1 & \cdot & \cdot \\ & & \cdot & \cdot \\ \cdot & \cdot & \cdot & \cdot \\ \cdot & \cdot & \cdot & \cdot \\ . & . & . & . \\ 2 & 1 & 0 & \ldots & 0\end{array}\right)$

3.2.7 The Matrices $F_{n}$. These $n \times n$ matrices are as follows

$$
F_{n}=\left(\begin{array}{cccc}
1 & 0 & \ldots & 0 \\
0 & 2 & \cdot & \cdot \\
\cdot & \cdot & \cdot & \cdot \\
\cdot & \cdot & \cdot & \cdot \\
\cdot & \cdot & \cdot & 0 \\
0 & \ldots & 0 & n
\end{array}\right)
$$


3.2.9. The matrices $H_{p, n}(t)$ and $\bar{H}_{p, n}(s)$. To define these matrices we need a number of nonalgebraic functions of $t$ and $s$. We shall take

$$
\begin{aligned}
& y_{1}(t)= \begin{cases}t & \text { for }|t| \leq 1 \\
t^{-1} & \text { for }|t| \geq 1\end{cases} \\
& y_{2}(t)=\exp \left(-t^{2}\right)
\end{aligned}
$$

$$
\begin{aligned}
& x_{1}(s)= \begin{cases}1 \text { for }|s| \leq 1 \\
s^{-2} \text { for }|s| \geq 1\end{cases} \\
& x_{2}(s)=\left\{\begin{array}{cc}
s^{-1} \exp \left(-s^{-2}\right) \text { for } s \neq 0 \\
0 & \text { for } s=0
\end{array}\right.
\end{aligned}
$$

Note that for $s \neq 0$

$$
x_{1}(s)=s^{-1} y_{1}\left(s^{-1}\right), x_{2}(s)=s^{-1} y_{2}\left(s^{-1}\right)
$$

The precise form of these functions is not important provided they are continuous, satisfy conditions (3.2.14) for $s \neq 0$, and are such that $y_{2}(t) \neq 0$ for all $t$ and $x_{1}(s) \neq 0$ for all $s$. It is perfectly possible to find $C^{\infty}$-functions satisfying these conditions (simply smooth the corners in these functions) but there are no polynomials in $t, s$ which satisfy these conditions.

We can now define the matrices $H_{p, n}(t)$ and $\bar{H}_{p, n}(s)$. Below we only give the $H_{p, n}(t)$. In each case $\bar{H}_{p, n}(s)$ is obtained from $H_{p, n}(t)$ by replacing $y_{1}(t)$ with $x_{1}(s)$ and $y_{2}(t)$ with $x_{2}(s)$.

(3.2.15) If $n=1, p \geq 2 \quad H_{1, n}(t)=\left(\begin{array}{c}y_{1}(t) \\ y_{2}(t) \\ 0 \\ \cdot \\ \cdot \\ \cdot \\ 0\end{array}\right)$

(3.2.16) If $n \geq 2, p>n \quad H_{p, n}(t)=\left(\begin{array}{ccccc}y_{1}(t) & 1 & 0 & \ldots & 0 \\ y_{2}(t) & 1 & 0 & \ldots & 0 \\ 0 & 1 & 0 & \ldots & 0 \\ 0 & 0 & . & \vdots \\ \cdot & . & . & . & \vdots \\ . & . & . & \cdot & 0 \\ 0 & 0 & \ldots & 0 & 1 \\ 0 & \ldots & \ldots & \ldots & 0 \\ \cdot & & & & \cdot \\ \cdot & & & & \cdot \\ . & & & & . \\ 0 & \ldots & \ldots & 0\end{array}\right) n-1$ 
(3.2.17) If $n \geq 2, p=n \quad H_{p, n}(t)=\left(\begin{array}{ccccc}y_{1}(t) & 1 & 0 & \ldots & 0 \\ y_{2}(t) & 1 & \cdot & \cdot \\ 0 & 0 & \cdot & \cdot \\ \cdot & \cdot & \cdot & \cdot \\ \cdot & \cdot & \cdot & \cdot \\ \cdot & \cdot & \cdot & \cdot \\ 0 & 0 & \underbrace{0 \ldots}_{n-2} & . & 0\end{array}\right) p-2$

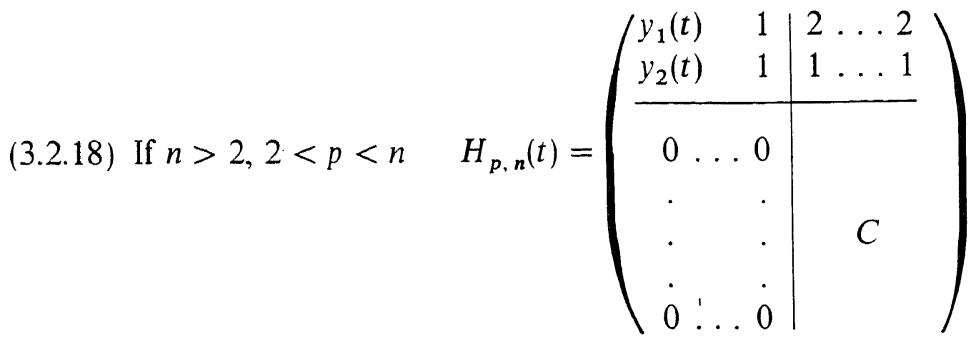

where $C$ is an $(p-2) \times(n-2)$ matrix independent of $t$ such that the rows of $C$ and the rowvector $(1, \ldots, 1) \in \mathbf{R}^{n-2}$ span a $(p-1)$ dimensional subspace of $\mathbf{R}^{n-2}$. (Such a $C$ exists because $2<p<n$ ).

(3.2.19) If $n>2, p=2 \quad H_{p, n}(t)=\left(\begin{array}{lll}y_{1}(t) & 1 & 2 \ldots 2 \\ y_{2}(t) & 1 & 1 \ldots 1\end{array}\right)$

3.3 An embedding $\mathbf{P}^{1}(\mathbf{R}) \rightarrow M_{m, n, p}^{c r, c o}(\mathbf{R})$. We now use the matrices defined in 3.2 above to define an embedding $\tau_{n, m, p}: \mathbf{P}^{1}(\mathbf{R}) \rightarrow \mathbf{M}_{m, n, p}^{c r}(\mathbf{R})$ for all $(n, m, p)$ such that $m>1$ and $p>1$, where $\mathbf{P}^{1}(\mathbf{R})$ is one dimensional real projective space (which is homeomorphic to the circle).

Let $m>1, p>1$. We define

$$
\begin{gathered}
\sigma_{n, m, p}: \mathbf{R} \rightarrow L_{m, n, p}(\mathbf{R}), t \mapsto\left(F_{n}, G_{n, m}(t, 1), H_{p, n}(t)\right. \\
\bar{\sigma}_{n, m, p}: \mathbf{R} \rightarrow L_{m, n, p}(\mathbf{R}), s \mapsto\left(F_{n}, G_{n, m}(1, s), \bar{H}_{p, n}(s)\right)
\end{gathered}
$$

Let $\alpha$ be the nice selection $\alpha=\{(0,2),(1,2), \ldots,(n-1,2)\}$ and let $\beta$ be the nice selection $\beta=\{(0,1),(1,1), \ldots,(n-1,1)\}$. One easily checks that (3.3.3)

$$
\sigma_{n, m, p}(\mathbf{R}) \subset U_{\alpha}^{c o}, \bar{\sigma}_{n, m, p}(\mathbf{R}) \subset U_{\beta}^{c o}
$$

Let $T(s)$ be the $n \times n$ matrix

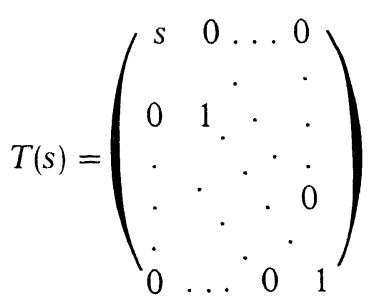

then we see that

$$
t s=1 \Rightarrow \sigma_{n, m, p}(t)^{T(s)}=\bar{\sigma}_{n, m, p}(s)
$$


To see this use the relations (3.2.14). Thus the composed continuous maps

$$
\begin{aligned}
& \mathbf{R} \stackrel{{ }_{n, m, p}}{\longrightarrow} L_{m, n, p}^{c r, c o}(\mathbf{R}) \rightarrow M_{m, n, p}^{c r . c o}(\mathbf{R}) \\
& \mathbf{R} \stackrel{\stackrel{\sigma_{n, m, p}}{\longrightarrow}}{\longrightarrow} L_{m, n, p}^{c r . c o}(\mathbf{R}) \rightarrow M_{m, n, p}^{c r . c o}(\mathbf{R})
\end{aligned}
$$

combine to define a continuous map

$$
\tau_{n, m, p}: \mathbf{P}^{1}(\mathbf{R}) \rightarrow M_{m, n, p}^{c r, c o}(\mathbf{R})
$$

3.3.6. Remark. One easily checks that $\left(F_{n}, G_{n, m}(t, 1), H_{p, n}(t)\right)$ and $\left(F_{n}, G_{n, m}(1, s)\right.$, $\left.H_{p, n}(s)\right)$ are $G L_{n}(\mathbf{R})$ equivalent if and only if $t s=1$. It follows that $\tau_{n, m, p}$ is in fact an embedding.

3.4. Some Remarks on Principal $G L_{n}(\mathbf{R})$-bundles. Let $\pi: E \rightarrow B$ be a principal $G L_{n}(\mathbf{R})$-bundle. Such a bundle can be described by giving an open covering $\left\{V_{a}\right\}$ of $B$ and transition functions

$$
\phi_{a b}: V_{a} \cap V_{b} \rightarrow G L_{n}(\mathbf{R})
$$

which statisfy

$$
\phi_{b c}(x) \phi_{a b}(x)=\phi_{a c}(x), x \in V_{a} \cap V_{b} \cap V_{c}
$$

Cf. Steenrod [4], 2.3 and 3.2.

3.4.3 Induced Bundles. Now let $f: B^{\prime} \rightarrow B$ be a continuous map. Let $V_{a}^{\prime}$ $=f^{-1}\left(V_{a}\right)$. One now defines transition functions $\phi_{a b}^{\prime}$ as the composite

$$
V_{a}^{\prime} \cap V_{h}^{\prime} \stackrel{f}{\rightarrow} V_{a} \cap V_{b} \stackrel{\phi}{\rightarrow} G L_{n}(\mathbf{R})
$$

These functions satisfy (of course) the analogue of (3.4.2) and hence define a principal (locally trivial) $G L_{n}(\mathbf{R})$-bundle $f: \pi: f ! E \rightarrow B^{\prime}$ over $B^{\prime}$. We remark that $f: \pi$ : $f: E \rightarrow B^{\prime}$ is trivial (i.e. isomorphic to the product bundle $G L_{n}(\mathbf{R}) \times B^{\prime} \rightarrow B^{\prime}$ ) if $\pi: E$ $\rightarrow B$ is trivial.

3.4.5. A second construction which we shall use is the following. The composed maps

$$
V_{a} \cap V_{b} \stackrel{\text { 中ub }}{\rightarrow} G L_{n}(\mathbf{R}) \stackrel{\text { der }}{\rightarrow} G L_{1}(\mathbf{R})
$$

define a set of transition functions with values in $G L_{1}(\mathbf{R})$ on $B$. These define a locally trivial principal $G L_{1}(\mathbf{R})$ bundle over $B$ which is of course trivial if the original bundle was trivial.

3.4.7. Example of a Nontricial Locally Trivial Principal $G L_{1}(\mathbf{R})$-Bundle. Consider $\mathbf{P}^{1}(\mathbf{R})=\{(t: s) \mid t \neq 0, s \neq 0\}$. Let $V_{1}=\{(t: 1)\}=\mathbf{R}$ and $V_{2}=\{(1: s)\}$ $=\mathbf{R}$. Then $V_{1} \cup V_{2}=\mathbf{P}^{1}(\mathbf{R})$. We now define a transition function

$$
\phi: V_{1} \cap V_{2} \rightarrow G L_{1}(\mathbf{R}),(t: s) \rightarrow t s^{-1}
$$

One checks easily that this bundle is non trivial (by showing that there is no 
continuous section). As a matter of fact the associated line segment bundle is the Mobius band bundle over the circle $\left(=\mathbf{P}^{1}(\mathbf{R})\right)$, cf. [4] example 1.3.

3.5. On the Nonexistence of Continuous Canonical Forms. We consider the continuous map $\tau_{n, m . p}: \mathbf{P}^{1}(\mathbf{R}) \rightarrow M_{m, n, p}^{c r}(\mathbf{R})$ constructed in section 3.3 above. Let $\alpha$ and $\beta$ be the selections defined just above (3.3.3), and let $V_{1}$ and $V_{2}$ be as in 3.4.7. Then we have from (3.3.3) that

$$
\tau_{n, m . p}\left(V_{1}\right) \subset V_{\alpha}^{\prime}, \tau_{n, m, p}\left(V_{2}\right) \subset V_{\beta}^{\prime}
$$

The transition function $U_{\alpha} \cap U_{\beta} \rightarrow G L_{n}(\mathbf{R})$ of the bundle $L_{m . n, p}^{c r}(\mathbf{R}) \rightarrow M_{m, n, p}^{c r}(\mathbf{R})$ is (according to (2.6.5))

$$
\rho_{\alpha \beta}(x)=R\left(F_{\alpha}\left(\psi_{\alpha}^{\prime-1}(x)\right), G_{\alpha}\left(\psi_{\alpha}^{\prime-1}(x)\right)\right)_{\beta}^{-1}
$$

By the definition of $\psi_{\alpha}^{\prime}(\mathrm{cf} .2 .5 .5)$ we see that $R\left(F_{\alpha}\left(\psi_{\alpha}^{\prime-1}(x)\right), G_{\alpha}\left(\psi_{\alpha}^{\prime-1}(x)\right)\right)$ can be calculated as follows. Take any $(F, G, H) \in U_{\alpha}$ such that $\pi(F, G, H)=x$. Then (cf. also (2.6.4))

$$
R\left(F_{\alpha}\left(\psi_{\alpha}^{\prime-1}(x)\right), G_{\alpha}\left(\psi_{\alpha}^{\prime-1}(x)\right)\right)=R(F, G)_{\alpha}^{-1} R(F, G)
$$

We now construct a $G L_{1}$-bundle over $\mathbf{P}^{1}(\mathbf{R})$ by first pulling back $L_{m . n . p}^{\text {cr }}(\mathbf{R})$ $\rightarrow M_{m, n, p}^{c r}(\mathbf{R})$ by means of $\tau_{n, m, p}$ (cf. 3.4.3) and then using determinants as in 3.4.6. It now follows from (3.5.3), (3.5.2) and (3.5.1) that the resulting bundle is given by the transition function

$$
V_{1} \cap V_{2} \rightarrow G L_{1}(\mathbf{R})
$$

$$
(t: 1) \mapsto \operatorname{det}\left(R\left(F_{n}, G_{n, m}(t, 1)_{\beta}^{-1}\right) \operatorname{det} R\left(F_{n}, G_{n, m}(t, 1)\right)_{\alpha}\right.
$$

An easy calculation shows that we find

(3.5.5) $V_{1} \cap V_{2} \rightarrow G L_{1}(\mathbf{R}),(t: 1) \mapsto t^{-1}$ if $n=1,(t: 1) \mapsto 2^{-n+2} t^{-1}$ if $n \geq 2$.

This is a nontrivial bundle. In fact the bundle defined by this transition function is isomorphic to the bundle defined in 3.4.7.

3.5.6. COROLLARY. The principal $G L_{n}(\mathbf{R})$-bundle $L_{m, n, p}^{c r}(\mathbf{R}) \rightarrow M_{m, n, p}^{c r}(\mathbf{R})$ is nontrivial.

3.5.7. In fact the examples show more: if $L^{\prime}$ is any $G L_{n}$-invariant subspace of $L_{n, m, p}$ and $M^{\prime}=\pi\left(L^{\prime}\right)$ and $\tau_{m, n, p}\left(\mathbf{P}^{1}(\mathbf{R})\right) \subset M^{\prime}$, then we have that the induced bundle $L^{\prime} \stackrel{\pi^{\prime}}{\rightarrow} M^{\prime}$ is nontrivial. [It also follows of course (because the pullback of a vector bundle by a homotopically trivial map is necessarily trivial) that the first homotopy group $\left.\pi_{1}\left(M^{\prime}\right) \neq 0\right]$.

Let $M_{m, n, p}^{w}(\mathbf{R})=\pi\left(L_{m, n, p}^{w}(\mathbf{R})\right)$ where $w$ is one of the following groups of symbols

$$
\text { cr cr,co } \quad c r, c o, \hat{\lambda} \quad \rho \quad \rho, \hat{\lambda}
$$

Then one easily checks from (3.2.2)-(3.2.6), (3.2.8), (3.2.15)-(3.2.19) and (3.2.11), (3.2.12) that:

\subsubsection{L.EMMA.}

(i) $\tau_{m . n . p}\left(\mathbf{P}^{1}(\mathbf{R})\right) \subset M_{m . n . p}^{w}(\mathbf{R})$ for all $w$ from the list (3.5.8) if $m \neq 1, n$ and $p \neq 1, n$.

(ii) $\tau_{m . n . p}\left(\mathbf{P}^{1}(\mathbf{R})\right) \subset M_{m . n . p}^{\text {cr.co. }}(\mathbf{R})$ if $m \neq 1$ and $p \neq 1$. 
3.5.10. COROLLARY. The principal $G L_{n}(\mathbf{R})$-bundles $L_{m, n, p}^{w}(\mathbf{R}) \rightarrow M_{m, n, p}^{w}(\mathbf{R})$ are nontrivial for all $w$ from the list 3.5.8 if $m \neq 1, n$ and $p \neq 1, n$. The principal bundles $L_{m, n, p}^{c r, c o, \lambda}(\mathbf{R}) \rightarrow M_{m, n, p}^{c r, c o, \lambda}(\mathbf{R})$ and $\left.L_{m, n, p}^{c r, c o}(\mathbf{R}) \rightarrow M_{m, n, p}^{c r, c o}(\mathbf{R})\right)$ are nontrivial if $m \neq 1$ and $p \neq 1$.

3.5.11. Proof. of Theorem 1.7. The only if parts of (i), (iii), (iv), (v), (vi) follow from corollaries 3.5.6 and 3.5.10 combined with lemma 3.1.2, except when $p=1$ in case (i). In this case one proceeds exactly as before starting with slightly modified maps $\sigma_{n, m, 1}^{1}, \bar{\sigma}_{n, m, 1}^{1}$ defined as in (3.3.1), (3.3.2) above except that the $H$ components are taken to be identically zero. The only if part of (ii) follows from the only if part of (i) by duality. Cf. 3.1.5 and 3.1.6. The if parts of (i), (ii) and (v) are proved by 3.1 .4 and 3.1.7. The if parts of (iii), (iv), (vi) follow from these because we have the inclusions

$$
\begin{aligned}
L_{m, n, p}^{c o, c r, \lambda}(\mathbf{R}) \subset L_{m, n, p}^{c o, c r}(\mathbf{R}) & c^{L_{m, n, p}^{c r}(\mathbf{R})} \\
L_{m, n, p}^{\rho, \lambda}(\mathbf{R}) \subset L_{m, n, p}^{\rho}(\mathbf{R}) . & \square^{L_{m, n, p}^{c o}(\mathbf{R})}
\end{aligned}
$$

\section{Families of Linear Dynamical Systems and the Fine Moduli Space $M_{m, n, p}^{c r}(\mathbf{R})$}

We have seen that as a rule continuous canonical forms cannot exist even on such a relatively small subspace as $L_{m, n, p}^{\rho, \lambda}(\mathbf{R})$. This section is devoted to showing that the next best thing is true: $M_{m, n, p}^{c r}(\mathbf{R})$ is a fine moduli space for a suitable notion of "families of linear dynamical systems".

\subsection{Families of Linear Dynamical Systems.}

4.1.1. Definition. A Family of Linear Dynamical Systems of Dimensions ( $n, m$, p) over a Topological Space $S$ consists of

(i) an $n$-dimensional vector bundle $p: E \rightarrow S$ over $S$.

(ii) a vectorbundle endomorphism $F: E \rightarrow E$

(iii) a vectorbundle homomorphism $G: S \times \mathbf{R}^{m} \rightarrow E$

(iv) a vectorbundle homomorphism $H: E \rightarrow S \times \mathbf{R}^{p}$

4.1.2 The Canonical Map Associated to a Family with Trivial Underlying Bundle. Let $\Sigma=(E, F, G, H)$ be a family of dynamical systems over $S$ such that $E$ is isomorphic to the trivial vectorbundle over $S$. Then we can find continuous sections $e_{1}, \ldots, e_{n}: S \rightarrow E$ such that $\left\{e_{1}(s), \ldots, e_{n}(s)\right\}$ is a basis for $E_{s}=p^{-1}(s)$ for all $s \in S$. Let $e_{1}^{\prime}, \ldots, e_{m}^{\prime}: S \rightarrow S \times \mathbf{R}^{m} ; e_{1}^{\prime \prime}, \ldots, e_{p}^{\prime \prime}: S \rightarrow S \times \mathbf{R}^{p}$ be the obvious "basis vector sections" of the trivial bundles $S \times \mathbf{R}^{m}$ and $S \times \mathbf{R}^{p}$. The vectorbundle homomorphisms $F, G, H$ induce homomorphisms $G_{s}: s \times \mathbf{R}^{m} \rightarrow E_{s}, F_{s}: E_{s} \rightarrow E_{s}, H_{s}$ : $E_{s} \rightarrow s \times \mathbf{R}^{p}$. Let $G(s, e), F(s, e), H(s, e)$ denote the matrices of these homomorphisms relative to the bases $\left\{e_{1}^{\prime}(s), \ldots, e_{m}^{\prime}(s)\right\},\left\{e_{1}(s), \ldots, e_{n}(s)\right\}$, $\left\{e_{1}^{\prime \prime}(s), \ldots, e_{p}^{\prime \prime}(s)\right\}$. Then because the sections $e_{1}^{\prime}, \ldots, e_{m}^{\prime} ; e_{1}, \ldots e_{n} ; e_{1}^{\prime \prime}, \ldots, e_{p}^{\prime \prime}$ are all continuous we find a continuous map.

$$
S \rightarrow L_{m, n, p}(\mathbf{R}), s \mapsto(F(s, e), G(s, e), H(s, e))
$$


which (obviously) depends on the choice of the sections $e_{1}, \ldots, e_{n}$. If $\bar{e}_{1}, \ldots, \bar{e}_{n}$ is a different set of $n$ sections of $E$ (and if we keep $e_{i}^{\prime}, e_{j}^{\prime \prime}$ as before) then the continuous map $s \mapsto(F(s, \bar{e}), G(s, \bar{e}), H(s, \bar{e}))$ is such that $(F, s, e), G(s, e), H(s, e))$ is $G L_{n}(\mathbf{R})$-equivalent to $(F(s, \bar{e}), G(s, \bar{e}), H(s, \bar{e})$ for all $s \in S$.

This means that the composite map

$$
f_{\Sigma}: S \rightarrow L_{m, n, p}(\mathbf{R}) \rightarrow M_{m, n, p}(\mathbf{R})
$$

is independent of the choice of the sections $\left\{e_{1}, \ldots, e_{n}\right\}$. We denote this continuous map with $f_{\Sigma}$. Informally we can say

4.1.5. Description of $f_{\Sigma}$. The family $(E, F, G, H)=\Sigma$ defines a "lineardynamical-system-up-to- $G L_{n}(\mathbf{R})$-equivalence" $\left(E_{s}, F_{s}, G_{s}, H_{s}\right)$ over every $s \in S ; f$ maps $s \in S$ to the point of $M_{m, n, p}(\mathbf{R})$ corresponding to this orbit.

4.1.6. The Canonical Map Associated to a Family. Now let $\Sigma=(E, F, G, H)$ be any family over $S$. There is an open covering $\left\{U_{a}\right\}$ of $S$ such that $E \mid U_{a}$ is trivial for all $a$. Thus by 4.1 .2 we have continuous maps associated to the families $\Sigma \mid U_{a}$ $=\left(E\left|U_{a}, F\right| U_{a}, G\left|U_{a}, H\right| U_{a}\right)$

$$
f_{a}: U_{a} \rightarrow M_{m, n, p}(\mathbf{R})
$$

which satisfy the description 4.1.5. It follows that $f_{a}\left|U_{a} \cap U_{b}=f_{b}\right| U_{a} \cap U_{b}$ so that the maps $f_{a}$ combine to define a continuous map

$$
f_{\Sigma}: S \rightarrow M_{m, n, p}(\mathbf{R})
$$

which also satisfies the description of 4.1.5.

4.1.8. Definition. The family $\Sigma=(E, F, G, H)$ is said to be completely reachable iff $f_{\Sigma}(s) \subset M_{m, n, p}^{c r}(\mathbf{R})$.

This simply means that the linear-dynamical-systems-up-to- $G L_{n}(\mathbf{R})$ equivalence $\left(E_{s}, F_{s}, G_{s}, H_{s}\right)$ are all completely reachable.

4.1.9. Remark. Using the construction of 4.1 .2 above we see that a family of linear dynamical systems $\Sigma$ can be defined by giving

(i) a covering $\left\{U_{a}\right\}$ of $S$

(ii) continuous maps $\psi_{a}: U_{a} \rightarrow L_{m, n, p}(\mathbf{R})$

(iii) continuous maps $\phi_{a b}: U_{a} \cap U_{b} \rightarrow G L_{n}(\mathbf{R})$

such that the following conditions are satisfied

(iv) $\phi_{b c}(x) \phi_{a b}(x)=\phi_{a c}(x)$ for all $x \in U_{a} \cap U_{b} \cap U_{c}$

(v) $\quad \psi_{a}(x)^{\phi_{a b}(x)}=\psi_{b}(x)$ for all $x \in U_{a} \cap U_{b}$

A family is completely reachable iff $\psi_{a}(x) \in L_{m, n, p}^{c r}(\mathbf{R})$ for all $a$ and all $x \in U_{a}$. So, informally, a family is locally a continous map of $S$ into $L_{m, n, p}(\mathbf{R})$.

4.2. Description of Families of Linear Dynamical Systems by Transition Functions. Let $(E, F, G, H)=\Sigma$ be a family of linear dynamical systems over $S$. The bundle $E$ can be described by an open covering $\left\{V_{a}\right\}$ of $S$ and transition functions $\phi_{a b}: V_{a} \cap V_{b} \rightarrow G L_{n}(\mathbf{R})$ satisfying $\phi_{b c}(x) \phi_{a b}(x)=\phi_{a c}(x)$ for all $x \in V_{a} \cap V_{b} \cap V_{c}$. Cf. Steenrod [4]. The family $\Sigma$ is now defined by giving in addition vectorbundle homomorphisms

(4.2.1) $F_{a}: V_{a} \times \mathbf{R}^{n} \rightarrow V_{a} \times \mathbf{R}^{n}, G_{a}: V_{a} \times \mathbf{R}^{m} \rightarrow V_{a} \times \mathbf{R}^{n}, H_{a}: V_{a} \times \mathbf{R}^{n} \rightarrow V_{a} \times \mathbf{R}^{p}$ 
such that the following diagram of induced vectorbundle homomorphisms commutes

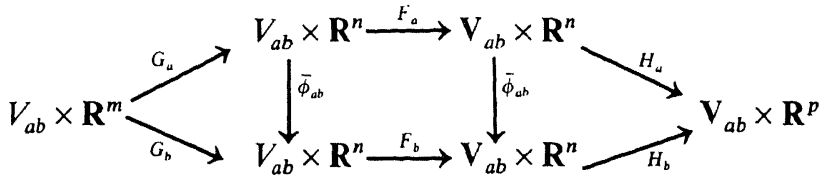

where $V_{a b}=V_{a} \cap V_{b}$ and $\bar{\phi}_{a b}(x, v)=\left(x, \phi_{a b}(x) v\right)$.

4.3. The Universal Family $\Sigma^{u}$ over $M_{m, n, p}^{c r}(\mathbf{R})$. We are now going to construct a certain very special completely reachable family $\Sigma^{u}$ over $M_{m, n, p}^{c r}(\mathbf{R})$. This family has the property that the induced linear-system-up-to- $G L_{n}(\mathbf{R})$-equivalence over $x \in M_{m, n, p}^{c r}(\mathbf{R})$ "is" the point $x$. To define the universal family $\Sigma^{u}$ we view $M_{m, n, p}^{c r}(\mathbf{R})$ as obtained by glueing together the pieces $V_{a}=\mathbf{R}^{m n+n p}$ by means of the isomorphisms $\phi_{\alpha \beta}$ of 2.5 above. That is we identify $V_{\alpha}$ with $V_{\alpha}^{\prime}$ for each $\alpha$ by means of the $\psi_{\alpha}^{\prime}$.

4.3.1. The Local Families $\Sigma_{a}$. Let $\alpha$ be a nice selection. For each $\alpha$ let $V_{\alpha}$ $=\mathbf{R}^{m n+n p}, E_{\alpha}=V_{\alpha} \times \mathbf{R}^{n}$ and let $p_{\alpha}: E_{\alpha} \rightarrow V_{\alpha}$ be the obvious projection. Let $\psi_{a}$ be the isomorphism $\psi_{\alpha}: V_{\alpha} \rightarrow W_{\alpha}$ of section 2.4 .5 above. We write $\psi_{\alpha}(x)=\left(F_{\alpha}(x)\right.$, $\left.G_{\alpha}(x), H_{\alpha}(x)\right)$. We now define the family $\Sigma_{\alpha}=\left(E_{\alpha}, F_{\alpha}, G_{\alpha}, H_{\alpha}\right)$ as follows

$$
\begin{aligned}
& F_{\alpha}: E_{\alpha} \rightarrow E_{\alpha},(x, v) \mapsto\left(x, F_{\alpha}(x) v\right) \\
& G_{\alpha}: V_{\alpha} \times \mathbf{R}^{m} \rightarrow E_{\alpha},(x, u) \mapsto\left(x, G_{\alpha}(x) u\right) \\
& H_{\alpha}: E_{\alpha} \rightarrow V_{\alpha} \times \mathbf{R}^{p},(x, v) \mapsto\left(x, H_{\alpha}(x) v\right)
\end{aligned}
$$

This defines a completely reachable family over $V_{\alpha}$ for all $\alpha$. The associated continuous map $f_{\Sigma_{x}}: V_{\alpha} \rightarrow M_{m, n, p}^{c r}(\mathbf{R})$ is the embedding $\psi_{\alpha}^{\prime}$ of lemma 2.5.5.

4.3.5. The (global) Family $\Sigma^{u}$. Now let $\beta$ be a second nice selection. Let $V_{\alpha \beta}, V_{\beta \alpha}$ and $\phi_{\alpha \beta}$ be as in section 2.5. Let $E_{\alpha \beta}=V_{\alpha \beta} \times \mathbf{R}^{n}, E_{\beta \alpha}=V_{\beta \alpha} \times \mathbf{R}^{n}$. We now define an isomorphism of vectorbundles $\phi_{\alpha \beta}: E_{\alpha \beta} \rightarrow E_{\beta \alpha}$ as follows

$$
\tilde{\phi}_{\alpha \beta}(x, v)=\left(\phi_{\alpha \beta}(x), R\left(F_{\alpha}(x), G_{\alpha}(x)\right)_{\beta}^{-1} v\right)
$$

It is obvious that $\tilde{\phi}_{\alpha \beta}$ induces an isomorphism of vectorspaces in each fibre and that the diagram

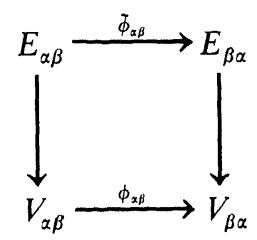

commutes. Also one readily checks that for all

$$
x \in V_{\alpha \beta \gamma}=\left\{x \in V_{\alpha} \mid \operatorname{det}\left(R\left(F_{\alpha}(x), G_{\alpha}(x)\right)_{\beta}\right) \neq 0 \text { and } \operatorname{det}\left(R\left(F_{\alpha}(x), G_{\alpha}(x)\right)_{\gamma}\right) \neq 0\right\}
$$

$$
\Phi_{\beta \gamma}\left(\Phi_{\alpha \beta}(x, v)=\tilde{\phi}_{\alpha \gamma}(x, v)\right.
$$

and that for all $x \in V_{\alpha \beta}$ 


$$
\begin{gathered}
\text { M. HAZEWINKEL } \\
\tilde{\phi}_{\alpha \beta}\left(F_{\alpha}(x, v)\right)=F_{\beta}\left(\tilde{\phi}_{\alpha \beta}(x, v)\right) \\
\tilde{\phi}_{\alpha \beta}\left(G_{\alpha}(x, u)\right)=G_{\beta}(x, u) \\
H_{\beta}\left(\tilde{\phi}_{\alpha \beta}(x, v)\right)=H_{\alpha}(x, v)
\end{gathered}
$$

Now let $E^{u} \rightarrow M_{m, n, p}^{c r}(\mathbf{R})$ be the $n$-vectorbundle obtained by glueing together the $E_{\alpha}$ by means of the $\tilde{\phi}_{\alpha \beta}$. Relations (4.3.9)-(4.3.11) then mean that $F_{\alpha}, H_{\alpha}, G_{\alpha}$ combine to define an endomorphism $F^{u}: E^{u} \rightarrow E^{u}$ and homomorphisms $G^{u}$ : $M_{m, n, p}^{c r}(\mathbf{R}) \times \mathbf{R}^{m} \rightarrow E^{u}$ and $H^{u}: E^{u} \rightarrow M_{m, n, p}^{c r}(\mathbf{R}) \times \mathbf{R}^{p}$, so that we have defined a completely reachable family $\Sigma^{u}$ over $M_{m, n, p}^{c r}(\mathbf{R})$. This family has the property: "the induced linear-system-up-to- $G L_{n}(\mathbf{R})$-equivalence over $x \in M_{m, n, p}^{c r}(\mathbf{R})$ is the point $x$ " because this is true for the local families $\Sigma_{\alpha}$.

4.3.12. Remark. From 4.3.6 (cf. also (3.5.3)) we see that $E^{u}$ is the $n$-vectorbundle associated to the principal $G L_{n}(\mathbf{R})$ bundle $L_{m, n, p}^{c r}(\mathbf{R}) \rightarrow M_{m, n, p}^{c r}(\mathbf{R})$.

4.4 The Functor $\Phi_{n, m, p}$ of Isomorphism Classes of Families of Linear Systems. Two families $\Sigma, \Sigma^{\prime}$ over a topological space $S$ are said to be isomorphic if there exists an isomorphism of vectorbundles $\phi: E \rightarrow E^{\prime}$ such that

$$
\phi F=F^{\prime} \phi, \phi G=G^{\prime}, H=H^{\prime} \phi
$$

For each topological space $S$ welet $\Phi_{n, m, p}^{c r}(S)$ denote the set of isomorphism classes of completely reachable families of linear systems (of dimensions $(n, m, p)$ ) over $S$. Now let $f: S^{\prime} \rightarrow S$ be a continuous map. By pulling everything back along $f$ we obtain a family $f: \Sigma=\left(f ! E, f^{\prime} F, f^{\prime} G, f^{\prime} H\right)$ over $S^{\prime}$ which is completely reachable if and only if $\Sigma$ is completely reachable. Informally $f: \Sigma$ is the family which induces over $s^{\prime} \in S^{\prime}$ the same linear system as $\Sigma$ induces over $f\left(s^{\prime}\right) \in S$. More precisely $f: \Sigma$ is defined as follows

$$
\begin{aligned}
& \left.f^{\prime} E=\left\{s^{\prime}, e\right) \in S^{\prime} \times E \mid f\left(s^{\prime}\right)=p(e)\right\} \\
& f^{\prime} F: f^{\prime} E \rightarrow f^{\prime} E,\left(s^{\prime}, e\right) \mapsto\left(s^{\prime}, F(e)\right) \\
& f^{\prime} G: S^{\prime} \times \mathbf{R}^{m} \rightarrow f^{\prime} E,\left(s^{\prime}, u\right) \rightarrow\left(s^{\prime}, G\left(f\left(s^{\prime}\right), u\right)\right) \\
& f^{\prime} H: f^{\prime} E \rightarrow S^{\prime} \times \mathbf{R}^{p},\left(s^{\prime}, e\right) \rightarrow\left(s^{\prime}, q H(e)\right)
\end{aligned}
$$

where $q: S \times \mathbf{R}^{p} \rightarrow S$ is the natural projection onto the first factor. If $\Sigma, \Sigma^{\prime}$ over $S$ are isomorphic families then $f^{\prime} \Sigma$ and $f^{\prime} \Sigma^{\prime}$ over $S^{\prime}$ are isomorphic. It follows that the pullback construction and the definition of $\Phi_{m, n, p}^{c r}(S)$ combine to define a functor

$$
\Phi_{n, m, p}^{c r}: \text { Top }^{o p p} \rightarrow \text { Set }
$$

where Top is the category of topological spaces and Set the category of sets.

4.4.7. Remark $\Phi_{n, m, p}^{c r}(p t)$, where $p t$ is the one point space is naturally the same as the underlying point set of $M_{m, n, p}^{c r}(\mathbf{R})$.

4.4.8 Let $\Sigma$ and $\hat{\Sigma}$ be two families of linear dynamical systems over $S$ defined relative the same covering $\left\{V_{a}\right\}$ of $S$ by transition functions $\phi_{a b}, \bar{\phi}_{a b}$ and local vectorbundle homomorphisms $F_{a}, \hat{F}_{a}, G_{a}, \hat{G}_{a}, H_{a}, \hat{H}_{a}$. Cf. 4.2 . Then $\Sigma$ and $\hat{\Sigma}$ are isomorphic families if and only if there exist continuous maps $\sigma_{a}: V_{a} \rightarrow G L_{n}(\mathbf{R})$ such that for all $a, b$ and $x \in V_{a} \cap V_{b}$ 


$$
\hat{\phi}_{a b}(x) \sigma_{a}(x)=\sigma_{b}(x) \phi_{a b}(x)
$$

and moreover the following diagram commutes for all $a$

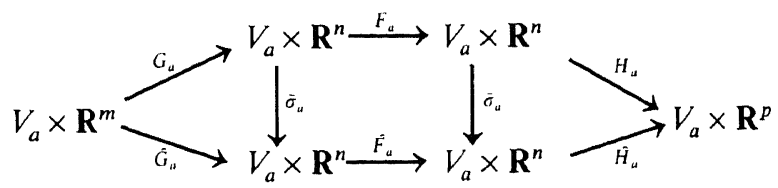

where $\bar{\sigma}_{a}(x, v)=\left(x, \sigma_{a}(x) v\right)$.

4.4.11. Let $\Sigma$ be a family over a topological space $S$ and let $f: S^{\prime} \rightarrow S$ be a continuous map. Suppose $\Sigma$ is given by means of a covering $\left\{V_{a}\right\}$ and local data $\phi_{a b}, F_{a}, G_{a}, H_{a}$ as in 4.2 above. Let $\left\{V_{\alpha}^{\prime}\right\}$ be any covering of $S^{\prime}$ which is finer than the covering $\left\{f^{-1}\left(V_{a}\right)\right\}$. For each $\alpha, \beta$ select an $a, b$ such that $f\left(V_{\alpha}^{\prime}\right) \subset V_{a}, f\left(V_{\beta}^{\prime}\right) \subset V_{b}$. Then the pullback family $f^{\prime} \Sigma$ can be described by the following data

$$
\begin{aligned}
& \phi_{\alpha \beta}^{\prime}: V_{\alpha}^{\prime} \cap V_{\beta}^{\prime} \rightarrow G L_{n}(\mathbf{R}), x \rightarrow \phi_{a b}\left(f^{\prime}(x)\right) \\
& F_{\alpha}: V_{\alpha}^{\prime} \times \mathbf{R}^{n} \rightarrow V_{\alpha}^{\prime} \times \mathbf{R}^{n},(x, v) \mapsto\left(x, F_{a, f(x)}(v)\right) \\
& G_{\alpha}: V_{\alpha}^{\prime} \times \mathbf{R}^{m} \rightarrow V_{\alpha}^{\prime} \times \mathbf{R}^{n},(x, u) \mapsto\left(x, G_{a, f(x)}(u)\right) \\
& H_{\alpha}: V_{\alpha}^{\prime} \times \mathbf{R}^{n} \rightarrow V_{\alpha}^{\prime} \times \mathbf{R}^{p},(x, v) \mapsto\left(x, H_{a, f(x)}(v)\right)
\end{aligned}
$$

where $F_{a, s}, G_{a, s}, H_{a, s}$ for $s \in V_{a}$ are defined by $F_{a}(s, v)=\left(s, F_{a, s}(v)\right), G_{a}(s, u)=(s$, $\left.G_{a, s}(u)\right), H_{a}(s, v)=\left(s, H_{a, s}(v)\right)$.

\subsection{The Fine Moduli Space $M_{m, n, p}^{c r}(\mathbf{R})$}

4.5.1. Definition. A fine moduli space for the functor $\Phi_{m, n . p}^{\mathrm{cr}}$ consists of a topological space $M$ and an isomorphism of functors $\mu: \Phi_{m . n, p}^{c r} \rightarrow \operatorname{Top}(-, M)$. I.e. $M$ represents the functor $\Phi_{m, n, p}^{c r}$.

4.5.2. THEOREM. $M_{m, n, p}^{c r}(\mathbf{R})$ is a fine moduli space for the functor $\Phi_{m, n, p}^{c r}$. More precisely the assignment $\Sigma \mapsto f_{\Sigma}$. induces a functorial isomorphism $\mu: \Phi_{m . n . p}^{\text {cr }}(S) \rightarrow \operatorname{Top}\left(S, M_{m, n, p}^{\text {cr }}(\mathbf{R})\right)$. The inverse isomorphism to $\mu$ assigns to $g: \mathbf{S} \rightarrow \mathbf{M}_{m . n . p}^{r r}(\mathbf{R})$ the isomorphism class of the family $g^{\prime} \Sigma^{u}$.

Proof. One checks easily that $f_{\Sigma}$ depends only on the isomorphism class of $\Sigma$ and that $\Sigma \mapsto f_{\Sigma}$ is functorial in the sense that $f_{g ! \Sigma}=f_{\Sigma} \cdot g$. This is most easily seen by using the description of $g^{\prime} \Sigma$ given in 4.4.11. We must now prove two things

(i) If $\Sigma=g^{\prime} \Sigma^{u}$, then $f_{\Sigma}=g$

(ii) $f_{\Sigma}^{!} \Sigma^{u}$ and $\Sigma$ are isomorphic families of linear systems.

To prove (i) it suffices to remark that

(iii) the system-up-to- $G L_{n}(\mathbf{R})$-equivalence $\Sigma_{s}$ over $s \in S$ is the system-up-to$G L_{n}(\mathbf{R})$-equivalence $\Sigma_{g(s)}^{u}(\mathrm{cf} .4 .4)$

(iv) the system-up-to- $G L_{n}(\mathbf{R})$-equivalence $\Sigma_{g(s)}^{u}$ "is" the point $g(s) \in M_{m, n, p}^{c r}(\mathbf{R})$ (cf. 4.3 just above 4.3.12).

(v) $f_{\Sigma}(s)$ is the point of $M_{m, n, p}^{c r}(\mathbf{R})$ representing the system-up-to- $G L_{n}(\mathbf{R})$ equivalence $\Sigma_{s}$. 
Alternatively one proves (i) by remarking that $f_{\Sigma^{u}}=i d$ (by (iv)) and by applying the formula $f_{g ! \Sigma}=f_{\Sigma} \circ g$.

It remains to prove (ii). Let $\Sigma$ be given by local data $\psi_{a b}, F_{a}, G_{a}, H_{a}$ relative a covering $\left\{V_{a}\right\}$ of $S$. By refining this covering if necessary we can assume that $\left\{V_{a}\right\}$ is finer than $\left\{f_{\Sigma}^{-1}\left(V_{\alpha}^{\prime}\right)\right\}$. For each $a, b, c$ let $\alpha, \beta$, $;$, be such that $f_{\Sigma}\left(V_{a}\right) \subset V_{\alpha}^{\prime}$ $f_{\Sigma}\left(V_{b}\right) \subset V_{\beta}^{\prime}, f_{\Sigma}\left(V_{c}\right) \subset V_{\gamma}^{\prime}$. Write $f$ for $f_{\Sigma}$. Then the pullback family $f^{\prime} \Sigma^{u}$ is given by the local data

$$
\begin{aligned}
\hat{\psi}_{a b}(x) & =R\left(F_{\alpha}(f(x)), G_{\alpha}(f(x))\right)_{\beta}^{-1} \\
\hat{F}_{a}(x, v) & =\left(x, F_{\alpha}(f(x)) v\right) \\
\hat{G}_{a}(x, u) & =\left(x, G_{\alpha}(f(x)) u\right) \\
\hat{H}_{a}(x, v) & =\left(x, H_{\alpha}(f(x)) v\right)
\end{aligned}
$$

Here we have identified $V_{\alpha}^{\prime} \subset M_{m, n, p}^{c r}(\mathbf{R})$ and $V_{\alpha}$ via $\psi_{\alpha}^{\prime}$, so that $\left(F_{\alpha}(x), G_{\alpha}(x), H_{\alpha}(x)\right)$ for $x \in V_{x}^{\prime}$ is the unique element in $W_{\alpha} \subset L_{m, n, p}^{c r}(\mathbf{R})$ which $\pi$ maps to $x$.

Let the family $\Sigma$ be given by the local data $\psi_{a b}, F_{a}, G_{a}, H_{a}$. Then by the definition of $f_{\Sigma}=f$ we know that the triples $\left(F_{a, s}, G_{a, s}, H_{a, s}\right)$ and $\left(F_{\alpha}(f(s)), G_{\alpha}(f(s))\right.$, $H_{\alpha}(f(s))$ are $G L_{n}(\mathbf{R})$-equivalent. This means that

$$
\left(F_{a, s}, G_{a, s}, H_{a, s}\right)^{T_{a}(s)}=\left(F _ { \alpha } \left(f(s), G_{\alpha}(f(s)), H_{\alpha}(f(s))\right.\right.
$$

for a certain $T_{a}(s) \in G L_{n}(\mathbf{R})$. In particular we have

$$
T_{a}(s) R\left(F_{a, s}, G_{a, s}\right)=R\left(F_{\alpha}(f(s)), G_{\alpha}(f(s))\right)
$$

so that we must have

$$
T_{a}(s)=R\left(F_{a, s}, G_{a, s}\right)_{\alpha}^{-1}
$$

(Note that (4.5.8) implies that $R\left(F_{a, s}, G_{a . s}\right)_{\alpha}$ has nonzero determinant). This defines a continuous map

$$
\sigma_{a}: V_{a} \rightarrow G L_{n}(\mathbf{R}), s \mapsto R\left(F_{a, s}, G_{a, s}\right)_{\alpha}^{-1}
$$

The local data $\psi_{a b}, F_{a}, H_{a}, G_{a}$ defining $\Sigma$ are related by the commutativity of the diagram 4.2.2 which in particular means that

$$
\psi_{a b}(s) R\left(F_{a, s}, G_{a, s}\right)=R\left(F_{b, s}, G_{b, s}\right)
$$

We shall now show that

$$
\hat{\psi}_{a b}(s) \sigma_{a}(s)=\sigma_{b}(s) \psi_{a b}(s)
$$

Indeed, we have using (4.5.8)-(4.5.10) and (4.5.3)

$$
\hat{\psi}_{a b}(s) \sigma_{a}(s) \boldsymbol{R}\left(F_{a, s}, G_{a, s}\right)=R\left(F_{\alpha}(f(s)), G_{\alpha}(f(s))\right)_{\beta}^{-1} R\left(F_{\alpha}(f(s)), G_{\alpha}(f(s))\right)
$$

On the other hand using (4.5.11) and (4.5.8)-(4.5.10)

$$
\sigma_{b}(s) \psi_{a b}(s) R\left(F_{a, s}, G_{a, s}\right)=\sigma_{b}(s) R\left(F_{b, s}, G_{b, s}\right)=R\left(F_{\beta}(f(s)), G_{\beta}(f(s))\right)
$$

which is equal to $R\left(F_{\alpha}(f(s)), G_{\alpha}(f(s))\right)_{\beta}^{-1} R\left(F_{\alpha}(f(s)), G_{\alpha}(f(s))\right)$ by the definition of the $F_{\alpha}, G_{\alpha}, F_{\beta}, G_{\beta}$. This proves (4.5.2) because $R\left(F_{a, s}, G_{a, s}\right)$ has rank $n$ (cf. 4.5.8). The commutativity of the diagram corresponding to (4.4.10) follows directly from (4.5.7) so that (by 4.4) the $\sigma_{a}$ do indeed define an isomorphism $\Sigma \rightarrow f_{\Sigma}^{\prime} \Sigma^{u}$. 
4.6. Remarks.

4.6.1. The underlying bundle $E^{u}$ of $\Sigma^{u}$ is the associated $\mathbf{R}^{n}$ bundle of the principal $G L_{n}(\mathbf{R})$ bundle $L_{m, n, p}^{c r}(\mathbf{R}) \rightarrow M_{m, n, p}^{c r}(\mathbf{R})$. So in particular it is nontrivial if $m \neq 1$. (Cf. Steenrod [4] 8.2-8.4). Also the restrictions of $E^{u}$ to various subspaces are often nontrivial. Cf. 3.5.10.

One can also use the fine moduli space property of $M_{m, n, p}^{c r}(\mathbf{R}), \Sigma^{u}$ to show that there exists a continuous canonical form on a suitable subspace $L^{\prime} \subset L_{m, n, p}^{c r}(\mathbf{R})$ iff the bundle $E^{u}$ restricted to $M^{\prime}=\pi\left(L^{\prime}\right)$ is trivial. Cf. [2] Thm 6.1.

\section{REFERENCES}

[1] M. Hazewinkel, R. E. Kalman, Moduli and Canonical Forms for Linear Dynamical Systems (to appear; a Preliminary Version of this is available as report 7504, Econometric Inst., Erasmus University, Rotterdam, 1975).

[2] M. Hazewinkel, R. E. Kalman, On Invariants, Canonical Forms and Moduli for Linear, Constant, Finite Dimensional, Dynamical Systems. In: Proc. CNR-CISM symposium on "Algebraic System Theory", Udine, 1975, Lect. Notes Economics Math. Syst. Theory 131, 48-60, Springer-Verlag, Berlin, Heidelberg, New York, 1976.

[3] R. E. Kalman, P. L. Falb, M. A. Arbib, Topics in Mathematical Systems Theory, McGraw-Hill, New York, 1969.

[4] N. E. STeEnrod, The Topology of Fibre Bundles, Princeton Univ. Press, Princeton, N.J., 1951.

[5] M. HAZEwinkel, Moduli and canonical Forms for Linear Dynamical Systems III: The algebraicgeometic case. (To appear. Proc. Inst./Sem. on Diff. Geometry for Control Engineers. Ames Research Centre (NASA), June/July 1976; a preliminary version is available as report 7610 , Econometric Inst., Erasmus Univ. Rotterdam), to be published: Math. Sci. Press.

[6] C. Byrnes, N. E. Hurt, On the Moduli of Linear Dynamical Systems, to appear, Advances in Mathematics.

Received June 30, 1976 and in revised form December 15, 1976. 